\title{
Development of High Throughput Assays for Establishing Process-Structure-Property Linkages in Multiphase Polycrystalline Metals: Application to Dual-Phase Steels
}

\author{
Ali Khosravani ${ }^{\mathrm{a}}$, Ahmet Cecen ${ }^{\mathrm{b}}$, and Surya R. Kalidindi ${ }^{\mathrm{a}^{*}}$ \\ ${ }^{a}$ Woodruff School of Mechanical Engineering, Georgia Institute of Technology, Atlanta, GA \\ 30332, USA. \\ ${ }^{\mathrm{b}}$ School of Computational Science \& Engineering, Georgia Institute of Technology, Atlanta, GA \\ 30332, USA \\ *Corresponding author
}

\begin{abstract}
Process-structure-property (PSP) linkages are central to the development and deployment of advanced materials in emerging technologies. Conventional approaches for establishing these are generally highly customized, qualitative, and demand major investments of time and effort. In this paper, we formulate novel, data-driven, high throughput, assays for exploring PSP linkages in structural metals. These assays are built on recent advances in low-dimensional quantification of material structure using spatial correlations and principal component analyses, as well as in the mechanical characterization using spherical nanoindentation. These novel protocols are demonstrated on a dual phase (DP) steel that exhibits rich multiphase polycrystalline microstructures.
\end{abstract}

Keywords: Spherical indentation; Process-Structure-Property Linkages; Dual phase steel; 2point spatial correlations. 


\section{Introduction}

A great deal of attention in materials innovation is now focused on the accelerated deployment of advanced materials in commercial products [1-6]. An important strategy for realizing this ambitious goal is the development, validation, and adoption of high throughput assays for rapid exploration of the extremely large materials and process design spaces involved. Indeed, significant effort in recent years [7-21] has been aimed at extracting high value (most useful for a selected application) process-structure-property (PSP) linkages [22-34] using a variety of approaches (including multiscale experiments, multiscale models, and data analytics).

Our focus in this paper will be on the protocols needed for the accelerated, experimental, exploration of PSP linkages in multiphase structural metals and alloys (e.g., dual-phase or DP steels). The processing histories employed on these materials typically involve a sequence of thermo-mechanical treatments, whereas the main properties (or performance characteristics) of interest are largely related to the plastic response of the material. Since one typically employs standard tensile tests [35] for evaluating the plastic properties of the material, it becomes necessary to make relatively large quantities of material samples with statistically homogeneous microstructures throughout the gage section (of the sample used in the tension test) for each combination of material chemistry and process path studied. This requirement drives up the cost and effort substantially, as is evident from some of the recent innovative efforts in this direction [12]. In other words, if it were possible to measure reliably the plastic response of the material in very small volumes, it would become possible to reduce dramatically the cost and effort involved in rapid screening of large design spaces in material chemistry and process histories.

Indentation has been employed extensively in prior literature [36-52] for evaluating the mechanical response of materials in small volumes. However, the use of this technique has been 
restricted largely to estimation of modulus and hardness. Only recently, it has been shown that it is possible to extract meaningful indentation stress-strain (ISS) curves directly from spherical indentation through a rigorous set of data analyses protocols. These new protocols have thus far been demonstrated mainly at the very small length scales (indentation zones much smaller than a single grain) [53-60]. There is, however, substantial promise for their application at the mesolength scales (where the indentation zone covers several grains [61]).

Beyond the measurement of mechanical response in small volumes, the next major impediment in extracting PSP linkages in advanced structural metals comes from the lack of a rigorous, yet practical, framework for the statistical quantification of their rich microstructures. Most of the currently employed approaches [38, 45, 47, 48, 51, 62-67] are based on highly simplified measures such as elemental compositions, phase volume fractions, average grain sizes of constituent phases, and the orientation distribution function (ODF). Clearly, the number of distinct microstructures one can reconstruct while meeting specific targets on these measures is extremely large, suggesting that this set of measures is likely to be inadequate for capturing all of the salient features of the microstructure. More formally, one might recognize most of the measures listed above (except the average grain size) as 1-point statistics of the microstructure in that they do not capture the morphological attributes of the microstructure. Indeed, one can employ the more advanced 2-point statistics (formally called the 2-point spatial correlations) [30, $31,68-75]$ to arrive at a more rigorous quantification of the material microstructure. However, most of the prior examples of the application of the framework of 2-point statistics have been limited to multiphase materials, with only a few focused on the application to polycrystalline materials [76-78]. The main difficulty of applying the 2-point statistics measures to polycrystalline samples arises from the very large number of local states present (for example, 
each distinct grain orientation encountered in the sample needs to be treated as a distinct local state), and the fact that the corresponding number of the distinct 2-point statistics is exceedingly large. In addition to being multiphase and polycrystalline, the microstructures of advanced alloys such as DP steels exhibit grain-scale heterogeneity in the spatial distribution of the dislocation densities (see Figure 1). Not surprisingly, a rigorous quantification of these complex microstructures is not readily accomplished with the currently available toolsets. Clearly, one needs to make suitable simplifications in order to establish practically useful PSP linkages for advanced structural metal alloys.

The main goal of this work is to explore the viability of high throughput experimental assays for establishing PSP linkages in multiphase polycrystalline metals, while utilizing small sample volumes and leveraging some of the recent advances described earlier (i.e., spherical microindentation stress-strain protocols and the framework of 2-point statistics). We have selected DP steels for this study because of their importance to several advanced technologies, owing to their excellent combination of continuous yielding behavior, high tensile strength, high work-hardening rate, and good ductility [38, 41, 45, 47, 51, 62, 63, 66, 79-87]. These properties are generally achieved in DP steels through a special heat treatment process called intercritical annealing $[36,52,64,65,67,80,84,88]$ during which the material is heated up to the austenite/ferrite region, held for a certain amount of time, and quenched to room temperature. Intercritical annealing results in formation of hard particles of martensite $\left(\alpha^{\prime}\right)$ in a soft matrix of ferrite $(\alpha)$ grains. This step is usually followed by additional cold work and heat treatment called bake hardening $(\mathrm{BH}[50,66,79,82,85-96]$; cf. Figure 1) to achieve the desired combination of mechanical properties. The main mechanisms involved in this overall thermomechanical process are as follows: (i) Introduction of mobile dislocations in ferrite grains at the vicinity of $\alpha / \alpha$ ' 
interfaces ([47, 62, 67, 79, 82, 83, 90, 97-99]; see Figure 1b), generally attributed to the volumetric plastic strains induced during the austenite to martensite transformation that occurs during the quenching from high temperature. This hypothesis is supported by transmission electron microscopy (TEM) observations [66, 85, 97, 98, 100] of higher densities of mobile dislocations in the ferrite matrix close to $\alpha / \alpha$ ' interfaces and the continuous yielding behavior exhibited by the DP steels. (ii) Formation of tangled networks of dislocations during the imposed plastic deformation, resulting in higher work hardening rates [79, 81, 85, 91, 97, 98] (see Figure 1c). (iii) Pining of dislocations, relieving residual stresses, and tempering martensite during the bake hardening process through diffusion of carbon atoms [66, 82, 83, 85, 86, 90-93, 95-97, 101] (see Figure 1d). As one might expect, the final properties exhibited by DP steel are critically dependent on the extent of the different mechanisms described above, which in turn depend critically on the process parameters employed in the production of the DP steel.

It is therefore clear that the process space (this is the set of all combinations of process parameters that could be utilized) to be explored in optimizing the set of final properties of interest in DP steels is extremely large. Consequently, high throughput assays mentioned earlier, if successfully employed, could produce dramatic acceleration in the deployment of these alloys in emerging technologies. In this work, we have formulated a foundational framework for the development of high throughput assays for establishing PSP linkages in advanced structural metals and alloys, and demonstrated its successful application to a selected process space in DP steels.

\section{Sample Prototyping}

The first step in the assays described in this paper is the high throughput prototyping of a sample library. Since our intent is to use indentation techniques for measuring plastic responses, 
we need to produce only small quantities of the material (no significant stipulations on sample shape either) for each selected material chemistry and/or process history. In this study, we decided to select a single chemical composition, and allow for variations in the thermomechanical processing history.

A four millimeter strip of low carbon steel with a chemical composition (in wt.\%) of $0.15 \mathrm{C}$, $1.4 \mathrm{Mn}, 0.04 \mathrm{P}, 0.04 \mathrm{~S}$ was secured and used in this study. The strip was cut into coupons of 10 $\mathrm{mm} \times 20 \mathrm{~mm} \times 4 \mathrm{~mm}$. All of the coupons were heat treated at $450{ }^{\circ} \mathrm{C}$ for 2 hours in order to produce an annealed microstructure, which then served as the starting material for the study described here. Table 1 (cf. Figure 1) summarizes the different processing histories included in this study, which were selected following the studies published in prior literature $[38,45,47,50$, $51,63-65,67,82,83,85,86,90,102-106]$. In the intercritical annealing process, the samples are heated to the specified temperature in a salt bath furnace (taking them into the austenite/ferrite region), held for 4 minutes, and quenched in an oil bath. Subsequent to the intercritical annealing, plane strain deformation was applied by rolling, and was followed by bake hardening at $170{ }^{\circ} \mathrm{C}$ for 20 minutes and quenching in water to the room temperature. As described in Table 1, three different intercritical annealing temperatures $\left(750,780\right.$, and $\left.810^{\circ} \mathrm{C}\right)$ and three amounts of total thickness reduction $(0,5$, and $10 \%)$ were selected for this study. This resulted in nine different processing conditions, with the sample labelling (see Table 1) capturing the processing history information. As an example, sample 750-05-170 indicates an intercritical annealing temperature of $750{ }^{\circ} \mathrm{C}$ and $5 \%$ thickness reduction, followed by bake hardening process at $170{ }^{\circ} \mathrm{C}$ for 20 minutes.

It is emphasized here that the time and effort expended in sample prototyping for the present study are minimal because the assays we intend to use do not pose stringent requirements on 
either the size or the shape of material volumes to be produced in each different processing history. In the present study, all of the sample prototyping was completed by one person in about 100 hours.

\section{Spherical Microindentation Stress-Strain Protocols}

As mentioned earlier, most of the prior work on indentation stress-strain curves has been focused on evaluating the plastic response at length scales that were significantly smaller than the grain size $[53,56,58-60,107]$. Only recently, these protocols have been extended to microindentation, where the length scales correspond to several grains (about 10-1000) in the indentation zone [108]. For microindentations conducted in the present study, we utilized a customized Zwick-Roell Z2.5 hardness tester with a $6.35 \mathrm{~mm}$ radius tungsten-carbide spherical tip. A constant crosshead speed of $0.1 \mathrm{~mm} / \mathrm{min}$ was utilized in all the tests reported here.

A significant challenge in extracting meaningful indentation stress-strain curves from the indentation experiments comes from the need to estimate reliably the evolving contact radius throughout the entire loading history [57, 109]. In modern nanoindenters (where the indentation zone sizes are typically much smaller than the grain size), this is accomplished through the CSM (continuous stiffness measurement) capability [109]. However, this capability does not yet exist for microindentation experiments. Consequently, the only way to estimate the contact radius reliably is to employ a multitude of unloading segments, and analyze them using Hertz theory [57]. Therefore, custom protocols were designed and implemented to allow a multitude of loading-unloading (corresponding to about 30-50\% of the peak force) cycles (see Figure 2(a)). Since each unloading segment produces one data point on the indentation stress-strain curve (Figure 2(b)), the number and frequency of the unloading segments has to be suitably adjusted to produce a meaningful and practically useful indentation stress-strain curve. 
The protocols for extracting indentation stress-strain curves from the raw load-displacement data from spherical indentation are based largely on Hertz theory (Eqs. 1-4) expressed as

$$
\begin{aligned}
& P=\frac{4}{3} E_{e f f} \sqrt{R_{e f f} h_{e}^{3}} \\
& \frac{1}{E_{e f f}}=\frac{1-v_{i}^{2}}{E_{i}}+\frac{1-v_{s}^{2}}{E_{s}} \\
& \frac{1}{R_{e f f}}=\frac{1}{R_{i}}+\frac{1}{R_{s}}
\end{aligned}
$$

where $P$ and $h_{e}$ are the indentation load and the elastic indenter displacement, and $R_{e f f}$ and $E_{e f f}$ are the effective radius and effective modulus, respectively. Constants $\left(E_{i}, v_{i}\right)$ and $\left(E_{s}, v_{s}\right)$ are Young's modulus and Poisson ratio for the indenter and the sample, respectively. For the initial elastic loading of a flat sample surface, the effective radius, $R_{e f f}$, is equal to the indenter radius, $R_{i}$. Therefore, it would be possible to extract $E_{\text {eff }}$ from the measured load-displacement using standard regression techniques; $E_{s}$ can be recovered using Eq. (2). The main hurdle in this protocol comes from having to make "zero-point" corrections to both measured load and measured displacement signals. However, with larger the indenter tips, the measurements are less sensitive to the surface quality of both sample and indenter. Consequently, for the microindentation testing here, there was no need for a zero-point load correction.

After the material in the indentation zone experiences plastic deformation, Eq. (1) needs to be modified to introduce the permanent displacement or residual height, $h_{r}$ :

$$
h=\left[\frac{3}{4} \frac{1}{E_{e f f}} \frac{1}{\sqrt{R_{e f f}}}\right]^{\frac{2}{3}} p^{\frac{2}{3}}+h_{r}
$$

Note that Eq. (4) can only be applied to unloading segments (these are assumed to be elastic). Applying standard regression techniques on the unloading segments to fit Eq. (4) produces reliable estimates of $h_{r}$ and $R_{e f f}$. In this analysis protocols, it is assumed that $E_{e f f}$ obtained 
from the initial elastic loading segment remains unchanged. This is a reasonable approximation for most materials, given the very small strains introduced in the indentation studies presented in this work. An unloading segment between $95 \%$ and $50 \%$ of the peak load (in each unloading) was employed in this work to estimate the evolving values of $h_{r}$ and $R_{e f f}$. After the initiation of plastic deformation in the sample, $R_{e f f}$ is no longer equal to $R_{i}$.

Indentation stress, $\sigma_{i n d}$, and indentation strain, $\varepsilon_{\text {ind }}$, corresponding to the start of each unloading segment are computed as

$$
\begin{gathered}
\sigma_{\text {ind }}=\frac{P_{\max }}{\pi a^{2}} \\
\varepsilon_{\text {ind }}=\frac{4}{3 \pi} \frac{h_{s, \max }}{a} \\
a=\sqrt{\boldsymbol{R}_{\text {eff }}\left(\boldsymbol{h}_{s, \text { max }}-\boldsymbol{h}_{r}\right)}
\end{gathered}
$$

where $P_{\max }$ and $h_{s, \max }$ are load and displacement at the peak of each unload, and $a$ is the contact radius. $h_{s, \max }$ is used to compensate the elastic displacement of the indenter, $h_{i}$, and is computed as

$$
\begin{gathered}
\boldsymbol{h}_{s}=\boldsymbol{h}-\boldsymbol{h}_{\boldsymbol{i}} \\
\boldsymbol{h}_{\boldsymbol{i}}=\frac{3\left(1-v_{i}^{2}\right) \boldsymbol{P}}{4 E_{i} a}
\end{gathered}
$$

Young's modulus, $E_{i}$, and a Poisson ratio, $v_{i}$, of $640 \mathrm{GPa}$ and 0.21 [110] were used for the 6.35 mm radius tungsten-carbide spherical indenter. Eq. (9) essentially estimates the indenter displacement by using Hertz's theory for indentation on a rigid flat surface.

An example indentation stress-strain curve produced in this work is plotted in Figure 2(b), which corresponds to sample 750-10-170. In this test, there were 20 unloading segments. The sample elastic modulus, $E_{s}$, is estimated from the initial elastic loading segment (shown 
highlighted in red in Figure 2(a)). The sample Young's modulus estimated from this indentation measurement is $207.8 \mathrm{GPa}$. The indentation yield strength defined based on $0.2 \%$ offset on the indentation stress-strain curve using an indentation modulus of $\frac{E_{S}}{1-v_{S}^{2}}$, was estimated to be 1191.2 MPa for this sample. Indentation results from the multiple tests (at least 10 tests on each sample) conducted in this work are summarized in Table 2. The average Young's modulus for all indentation measurements on all samples was $201.4 \mathrm{GPa}$ with a standard deviation of $14.2 \mathrm{GPa}$, which compares well with reported Young's modulus in literature for steel from conventional tensile testing [111]. It is important to recognize that the indentation zone in the experiments reported here is sufficiently large to include multiple microstructural features, including ferrite and martensite regions. This is illustrated in Figure 3 for sample 750-00-000, where the estimated indentation zone sizes are delineated both at yield and at the end of the indentation test. The solid line circle in Figure 3(a) shows the estimated indentation zone size at the end of test, and the dashed line circle corresponds to the zone size at the indentation yield point. The image on right, Figure 3(b), is an SEM image taken on the same sample, whose size was made to correspond to the contact area at indentation yield. As one can see, the indentation area is large enough to justify the measurements reported here as measurements of the bulk mechanical response of the sample, especially keeping in mind that multiple indentations are conducted on each sample and an average is reported for all indentations conducted on the sample (see Table 2). An estimate of the number of grains in the indentation zone can be obtained assuming a cylindrical primary indentation zone of radius $a$ and height $2.4 a$ [109]. The contact radii $a$ at the yield point are listed in Table 2 for all tests. Considering the average grain size $(30 \mu \mathrm{m})$ for the material tested and an average contact radius at yield in the range of 142-182 $\mu \mathrm{m}$ (see Table 2), the number of grains in the primary zone is estimated to be in the range of 190-400 grains. 
It is clear from Table 2 that the strength of the DP steel is largely controlled by the amount of both martensite and the imposed cold work. Increasing the amount of martensite (by means of quenching from higher intercritical annealing temperatures) results in strengthening of the DP

steel. For example, the indentation yield strength, $\sigma_{Y}^{\text {ind }}$, increases from $899.5 \pm 62.9 \mathrm{MPa}$ to $1168.6 \pm 186.8 \mathrm{MPa}$, when the quenching temperature is increased from $750^{\circ} \mathrm{C}$ to $810^{\circ} \mathrm{C}$. The same trends can be seen even after 5\% and $10 \%$ cold work and bake hardening were applied. These trends are consistent with the observed results in literature $[45,51,66,82,87,92,93,95$, 104]. Although, it is possible to establish suitable conversion factors to estimate uniaxial yield values from the indentation yield values [108], this was not pursued in the present work as the focus in this work is on the establishment of high throughput assays.

It is emphasized here that the sample preparation for the microindentation tests reported here is identical to the sample preparation used for microstructure characterization (described next). In other words, the same sample preparation is used for both aspects of the study, leading to substantial economy in both time and effort expended.

\section{Microstructure Characterization}

The next step in the assays employed in this work focused on microstructure characterization. In prior literature, the most common approaches employed in microstructure characterization of DP steels included optical microscopy [36, 38, 45, 51, 64-66, 83, 88, 104-106], scanning electron microscopy [36, 41, 42, 47, 51, 63, 88, 104, 106], and electron back-scattered diffraction (EBSD) $[47,48,112-116]$. While each of these techniques has demonstrated certain advantages over the others for highlighting specific aspects of the microstructure, our goal here is to identify and employ the protocols that give us the best chance at establishing reliable PSP linkages. 
Furthermore, we also desire to minimize the overall effort required so that we can maximize the potential for high throughput.

After a careful consideration of the relative merits of the different protocols, it was decided to employ the EBSD technique for the present study. In addition to mapping lattice orientations in ferrite regions and indirectly identifying martensite regions (based on very poor diffraction patterns), the EBSD technique provides critically needed information on the extent of deformation pinning (in the ferrite grains next to the martensite grains; cf. Figure 1) through an analysis of the lattice misorientations. Consequently, although the use of the EBSD technique represented a time and effort intensive option for the present study, it was deemed essential as the other microscopy techniques are incapable of capturing any relevant information on the extent of pinned dislocations in the samples. It is emphasized that some measure (even if it is an indirect measure) of the pinned dislocation density is essential to correlate to the plastic properties of the sample.

EBSD scans were carried out in a field emission gun scanning electron microscope at $20 \mathrm{kV}$. Samples were prepared (for both EBSD and indentation studies) using standard metallography protocols. This involved grinding with silicon carbide papers down to grade 4000, and polishing sequentially with diamond suspensions of $3 \mu \mathrm{m}, 1 \mu \mathrm{m}$, and OP-A alumina suspension. The final step in sample preparation was electro-polishing using A3 Struers electrolyte at $5{ }^{\circ} \mathrm{C}$.

Several scans (around 9 or 10) were collected on ND plane on all samples with $1 \mu \mathrm{m}$ resolution. It was also decided to keep the scan size consistent with the primary indentation zone in the mechanical characterization described earlier. The EBSD scan size was selected to be $400 \times 400 \mu \mathrm{m}^{2}$. This is important because one of the goals of this study is to find linkages between indentation yield strength, $\sigma_{Y}^{\text {ind }}$, and the microstructure. Just as we conducted multiple 
indentation measurements on each sample, we have also conducted multiple EBSD scans on each sample to allow for the quantification of variance.

The collected raw data was post-analyzed using TSL OIM $^{\odot}$ software. Example EBSD scans obtained for different samples in our sample library are shown in Figure 4. In these figures, multicolored regions represent ferrite grains whose lattice orientations have been mapped out with a $1 \mu \mathrm{m}$ spatial resolution and martensite regions are colored black. One can see that the martensite mostly forms at grain boundary regions and triple points. The amounts of martensite appear to increase with quenching from a higher intercritical temperature (from top row to the bottom row in Figure 4). Furthermore, a close inspection of the EBSD scans in Figure 4 reveals that samples subjected to higher deformation levels show significantly higher levels (and extent) of in-grain misorientations (i.e., compare left side maps with right side ones in Figure 4). The main implication here is that the misorientation fields in the samples do capture some information on the extent of pinned dislocations and their spatial distributions in the microstructure. This information will be utilized later in establishing the PSP linkages of interest in this study.

Figure 5 shows $\varphi_{2}=45^{\circ}$ section of the orientation distribution functions (ODFs) for all samples produced in this study. All textures depict $\alpha$ fiber, $\beta$ fiber, and cube components. Applying deformation by means of cold work moves the strongest texture component from the $\{001\}\langle 110\rangle \alpha$ fiber component to the $\{111\}\langle 110\rangle \beta$ fiber component. It is seen that the differences in texture between the samples are not very significant.

As it was mentioned earlier, EBSD technique was chosen for this study because it provides information about phase as well as the extent of pinned dislocations (as indicated by local orientation gradients in the ferrite). In the case of DP steel, martensite can be identified based on 
low values of CI (confidence index) and IQ (image quality) $[47,48,112,113,115,116]$. The poor quality of diffraction patterns in martensite is generally attributed to the high density of defects in these regions. More importantly, as the DP steel is deformed, dislocations accumulate at the martensite-ferrite boundaries producing significant local gradients in the lattice orientations (see the right column in Figure 4). In other words, a suitable measure of the local orientation gradients can serve as an indicator of the local (microscale) hardening in the sample.

Currently used measures of in-grain misorientations can be broadly grouped as either grainbased or kernel-based approaches. Grain based approaches such as GOS (grain orientation spread) [117-119], GAM (grain average misorientation) [117, 119] estimate an average value of misorientation based on all measurements within one grain. In contrast, in kernel based approaches such as KAM (kernel average misorientation) [99, 120-122], quantities such as local average misorientation and local misorientation spread are computed at each grid point in the measurement scan. Clearly, the KAM is a better indicator of the local hardening in the microstructure.

$\mathrm{KAM}$ is defined as the average misorientation between the orientation at the center of the kernel and all its neighbors (Figure 6(a)). In this approach, misorientations that exceed some tolerance value (maximum misorientation, $5^{\circ}$ ) are excluded from the averaging calculation, to avoid erroneous consideration of neighbors across a grain boundary. The distributions of KAM values recovered from the EBSD scans for the different samples in our sample library are shown in Figure 6(b).

For the quantification of each microstructure, we need to make a judicious selection of the local states to be included in the analyses. Local states denote attributes needed to assign the local properties associated with each voxel of the microstructure. For the samples studied, the 
local state would be ideally defined by a combination of the phase identifier (i.e., ferrite or martensite), the lattice orientation, the KAM value, among several other potential attributes. It should, however, be recognized that adding more local state attributes will exponentially increase the effort expended in arriving at PSP linkages of interest. Therefore, it behooves us to make smart choices in the selection of local states.

In the present study, we have decided to include only the phase identifier and the KAM as the local state attributes. Since the texture in the sample did not change appreciably between the different processing histories employed in the study, the lattice orientation was not included as a local state variable in each voxel. This is also partly because the inclusion of the lattice orientation as a state variable increases dramatically the effort involved in microstructure quantification [76, 78], which is not justified when there do not exist significant differences in crystallographic and/or morphological textures in the ensemble of samples studied. Finally, it was also decided to use a discretized representation of the KAM value in the microstructure quantification pursued in this work. Based on the histograms shown in Figure 6(b), it was decided that a KAM value of $0.8^{\circ}$ or more would be labelled as "deformed ferrite", while KAM values lower than $0.8^{\circ}$ would be labelled as "undeformed ferrite". Note that $95 \%$ of the KAM values in all samples quenched at 750,780 , and $810^{\circ} \mathrm{C}$ (without any cold work) exhibited values below $0.8^{\circ}$ (see Figure 6(b)), justifying the selection of this value.

As a consequence of the simplifications introduced above, each voxel in the microstructure is assigned to one of three discrete local states: martensite, deformed ferrite, or undeformed ferrite. Although one might raise objections to such gross simplifications, it is important to recognize and understand that a much more detailed quantification of the microstructure may actually be not warranted in the present study. Indeed, we will demonstrate that this simple selection of local 
states is adequate in the present study to establish reliable PSP linkages of interest. Of course, as one expands the processing windows to be included in the PSP linkages being formulated, one would need to add additional attributes in the description of the local states.

Figure 6(c)-(d) show selected areas of the measured IPF maps for 750-00-000 and 750-10-170 samples. Figure 6(e) and 6(f) show the corresponding discretized microstructures employed in the quantification of the material structure in this study. As noted earlier, these microstructures have only three discrete states, which are colored black, white, and blue in Figures 6(e) and 6(f). Note the increase in the amount of blue voxels, especially near the martensite interfaces, in the 750-10-170 microstructure compared to the 750-00-000 microstructure.

In this study, we will employ the framework of 2-point statistics to quantify the microstructures generated in this work. These are denoted as $f_{r}^{h h^{\prime}}$ and are computed as [123]

$$
f_{r}^{h h^{\prime}}=\frac{1}{S_{r}} \sum_{s=1}^{S} m_{S}^{h} m_{s+r}^{h \prime}
$$

where $S$ is the total number of bins in the microstructure, $m_{S}^{h}$ is discretized representation of the microstructure denoting the volume fraction of local state $h$ in voxel $s$, and $r$ indexes discretized vectors placed into the microstructure for evaluating the 2-point statistics. In essence, the 2-point spatial correlations capture the probability of finding discrete local states $h$ and $h^{\prime}$ at the tail and head of a discrete vector indexed by $r$. Since, the microstructures studied here have three local states, a total of nine 2-point spatial correlations can be defined. Three of the computed 2-point correlations for sample 780-10-170 are shown in Figure 7, along with the original EBSD scan and the discretized microstructure. The autocorrelation of the martensite (colored black), the autocorrelation of the deformed ferrite (colored blue), and the cross-correlation of the martensite and the deformed ferrite are shown in Figure 7(c), 7(d), and 7(e), respectively. These spatial statistics maps contain a tremendous amount of information about the morphology and 
distribution of the local states in the microstructure. For example, the center peaks in the autocorrelations provide the volume fraction information. Consequently, it can be seen that the volume fractions of martensite and deformed ferrite are 0.45 and 0.276 , respectively, for the microstructure shown in Figure 7. The contours in the center of the autocorrelation plots provide quantitative information on the shape of local state field in the microstructure. One can also extract a large number of conventional microstructure measures from these plots [30,31], and even reconstruct the original microstructure from its spatial correlations [124-126].

The quantification of microstructures using the framework of 2-point statistics produces a very large vector of descriptors for the microstructure (every voxel in each of the correlation maps would be an entry in this vector). Principal component analyses (PCA) has been shown to be remarkably effective in arriving at objective (data-driven) low dimensional representations $[34,68,74,127-131]$ of the microstructure. Mathematically, this can be expressed as

$$
f_{r}^{(k)}=\sum_{i=1}^{P-1} \alpha_{i}^{(k)} \varphi_{i r}+\tilde{f}_{r}
$$

where $f_{r}^{(k)}$ denotes a contracted representation (i.e., as a single vector) of all the spatial correlations of a microstructure indexed by $k, \tilde{f}_{r}$ denotes the ensemble average of all microstructures included in the analyses, $\varphi_{i r}$ denote the directions of the new linearly transformed reference frame (identified by PCA) and $\alpha_{i}$ are the coordinates (also called PC scores) in the new reference frame. A salient feature of PCA is that it transforms the data into a new orthogonal frame where the axes are ordered by the maximum variance seen in the data.

For the present case study, PCA was performed on a total of 81 EBSD scans (nine scans for each of nine process histories). The analyses indicated that only two PC scores (see Figure 8) are adequate to capture $99.4 \%$ of the variance between the different microstructures in this ensemble (i.e., all 81 scans included in the study). In other words, only two numbers (i.e., the first two PC 
scores denoted as PC1 and PC2) are adequate to distinguish each of the microstructures produced in this study. This is indeed a remarkable dimensionality reduction, and to the best of our knowledge, unmatched by any other existing framework for microstructure quantification.

For microstructures with three local states, only two of the nine 2-point spatial correlations would be independent [72], if the boundaries of the microstructure were treated as periodic. However, through repeated trials of the PCA described above, we observed that using the two autocorrelations and the cross-correlation shown in Figure 7 provided a good separation between the microstructures of each of the nine groups of samples.

Figure 9 shows all of the microstructures included in the present study in the low dimensional PC space, where each point represents one microstructure. The interclass (nine different processing histories) and the intraclass (nine microstructures from each sample) variances are clearly discernable in this plot. Furthermore, one should note that the variance in PC1 is higher than the variance in PC2 for the ensemble of microstructures studied. It is observed that the intraclass variance for the deformed samples is higher than the intraclass variance for the undeformed samples. Most importantly, one can discern trends in the microstructure evolution by following the centroids of each class of microstructures. For example, one can capture quantitatively the effect of quenching temperature by following the dashed lines. Similarly, one can follow the effect of cold work by following the solid lines in this figure. These trend lines can be called processing paths or processing streamlines, as they capture quantitatively the salient aspects of the influence of process parameters on the evolved microstructures. It is indeed remarkable that a simple data-driven approach involving 2-point spatial correlations and PCA can capture so much detail with just two PCs. 


\section{Extraction of PSP Linkages}

Building on the low dimensional structure representation obtained in the previous section (i.e., PC1 and PC2), we are now in a position to establish PSP linkages of interest. For the present case study, the process parameters are taken as the intercritical annealing temperature $(T)$ from which the samples are quenched, and the imposed thickness reduction during the rolling process $(D)$. The desired process-structure linkages are then established using least squares regression and cross-validation techniques [68], which resulted in a second-order polynomial fit expressed as

$P C 1=-5612.478+14.089 T-5.138 D+\left(15.308(D \times T)-8.862 T^{2}-332.461\right) \times 10^{-3}(12)$

$P C 2=-2734.355+6.665 T-1.682 D+\left(1.823(D \times T)-4.035 T^{2}-131.892\right) \times 10^{-3}$

The accuracy of these models is displayed as parity plots in Figure 10, where each point corresponds to one process history (i.e., the average of all 9 scans from each sample).

PC1 and PC2 denote the strengths of certain spatial patterns in the microstructure. These patterns are visualized in Figure 11. The first column in this figure shows the ensemble average, while the second and third columns depict the patterns captured by PC1 and PC2, respectively. In the PC space, each microstructure is approximated as a weighted deviation from the ensemble average (see Eq. (11)) using the $\varphi_{\text {ir }}$ (plotted in second and third columns of Figure 11) as an orthonormal basis. As an example, as PC1 is increased, it is seen (from the second column in Figure 11) that the volume fractions of martensite and deformed ferrite will both increase and the martensite regions will become more elongated in the horizontal direction. Likewise, when PC2 is increased, there is an increase in the volume fraction of martensite accompanied with a decrease in the volume fraction of the deformed ferrite, while making the martensite regions more equiaxed. Of course, there is a lot more information embedded in the basis maps shown in 
Figure 11, which actually contributes to the remarkable efficacy of PCA in arriving at the low dimensional representation employed in this study. There currently do not yet exist any systematic approaches for carefully studying these basis maps.

The approach described above can also be applied to establish a structure-property linkage. For the present case study, the recovered structure-property linkage is expressed as

$$
\sigma_{Y}^{i n d}=1156.4+9.2(P C 1)+5.5(P C 2)
$$

where $\sigma_{Y}^{i n d}$ denotes the yield strength of the material measured in the indentation protocols described earlier. The accuracy of this linkage is depicted in the parity plot shown in Figure 12.

The reader is reminded that the linkages developed here are data-driven. One of the implications is that the PSP linkages built here will change when new data is added. This is to be expected, especially since we used only nine different processing histories in this study. When a substantial amount of data becomes available, we could expect the PSP linkages to become stable (i.e., there would be no need to change the PSP linkages with the addition of new data points). This indeed is the most attractive feature of the protocols described here. They allow a coordinated effort by research groups and/or investigators in ways that leverage all available data and minimize redundant effort in establishing such linkages. Furthermore, with the use of high throughput strategies, such as the indentation methods described in this paper, it is conceivable to aggregate PSP linkages of high value to the scientific community at dramatically accelerated pace compared to the conventional approaches used currently.

\section{Discussion}

The case study described above points mainly to the tremendous potential for developing and implementing high throughput assays in the exploration of PSP linkages in structural alloys. It is fully acknowledged that the term "high throughput" is being used here to signify the acceleration 
when compared to conventional approaches. We now review the main elements of the envisioned high throughput assays, and identify specific opportunities for future development.

One of the key elements of the high throughput assays described in the paper is in the use of instrumented indentation techniques for local measurements of mechanical response and their expression as normalized stress-strain curves. Indeed, the expression of the local mechanical response in the form of stress-strain curves is critically important for ensuring reliability and reproducibility of the measurements. Furthermore, the ability to conduct a large number of these tests at different length scales (by simply changing the indenter tip radius) even in a single sample, is clearly of major significance to multiscale modeling efforts. At the present time, this is the most practical approach for producing the large amount of experimental observations needed to calibrate the numerous parameters in multiscale modeling efforts [132-134]. However, in order to realize its full potential, additional work is needed in automation of this technique across all length scales of interest. As mentioned earlier, the experimental protocols are already fairly automated for the indentations conducted at the very small length scales. However, the experimental protocols for the indentations conducted at the larger length scales (indentation zone sizes covering multiple grains) are yet to be automated. The indentation stress-strain analyses protocols used in this work have been coded and are being prepared for broad dissemination as an open source software. While this software speeds up the analyses significantly, it still needs further development and automation. One other significant benefit of the indentation protocols is that they allow for parallelization. Indeed, several of the commercial vendors for instrumented indenters are currently exploring concepts that allow for simultaneous indentation measurements on a grid (using multiple indenters simultaneously). Success in one or more of the concepts mentioned above will contribute significantly to the further development of the high throughput assays presented in this work. 
A second key element of the high throughput assays described here is in the use of the emerging data science techniques for formulating the reduced-order PSP linkages. The efficacy is derived from the ability to template the workflows in ways that they can be applied to a broad range of material systems over a multitude of length/structure scales [68, 127, 135-138]. However, the use of a common template does not imply lack of flexibility. On the contrary, the modular approach comprising a specific sequence of steps involving a (i) digital representation of the microstructure, (ii) evaluation of microstructure statistics, (iii) low dimensional representation of the microstructure, and (iv) extraction of a reduced-order model allows for the development of highly efficient (high performance) computer codes leading to a systematic evaluation and objective identification of the best protocols. This is because the modern data science approaches allow one to establish a large number of possible workflow pipelines (utilizing the best available codes), push a large amount of data through these pipelines in parallelized efforts, and disseminate the findings in open source and open access repositories. As examples, in the case study presented in this work, one could evaluate a series of other choices for the digital representation of the microstructure (e.g., use other measures of local lattice misorientation or even the full information of the lattice orientation in the local state descriptors employed, use information gathered by other microscopy techniques), microstructure statistics (e.g., use chord-length distributions [139] instead of 2-point statistics), low dimensional representations (e.g., factor analysis [140], projection pursuit [141], independent component analysis [142]), and machine learning approaches (e.g., M5 model trees [143], support vector machines [144]). A further complicating factor in arriving at the most robust and reliable PSP linkages comes from the fact that the models produced in this approach are, to a significant extent, dependent on how much data is available (this is why these have been referred as data-driven throughout this paper). In other words, one should expect the best protocols and the 
models to change as more data is added to the analyses (one can hope that the models stabilize after a critical amount of data has been employed - but in the interim one can still use the best available models at any time). Because of the very large number of choices one faces as well the significant cross-disciplinary expertise required for such a systematic evaluation, it is necessary to transition this pursuit from individuals, or even small groups of individuals, to a community-level curation effort. The data science workflows of the type presented in this work can lead this important transformation, and really accelerate the collection and curation of the PSP linkages for a broad range of materials systems of general interest to the entire materials science community $[32,33,145,146]$.

\section{Conclusions}

In this study, we have formulated and demonstrated novel high throughput assays for extracting data-driven, high value, PSP linkages in complex multiphase structural alloys such as DP steels. These new protocols utilized spherical indentation stress-strain measurements, 2-point spatial correlations, principle component analysis (PCA), and regression techniques. Following are the major findings from this study:

1. Spherical indentation is a reliable high throughput tool for probing the changes in the yield behavior of dual phase steel processed at different intercritical annealing temperatures and different amounts of cold work applied before bake hardening. While indentation elastic modulus showed only small variations as expected, indentation yield strength varied significantly with the processing history. This is attributed to the many changes induced in the microstructure of the sample with the processing history. These have included not only changes in the volume fractions of the different constituents, but also their spatial distributions. Additionally, the spatial 
distribution of the dislocation content at the martensite/ferrite interface also played an important role in controlling the final plastic yield values in the sample.

2. The application of PCA on the 2-point correlations provided a rigorous framework for objective, low-dimensional, quantification of the microstructures in DP steels. In this work, this protocol was applied only on the phase and misorientation information. The protocol found that only two PCs are needed to distinguish the microstructures studied and establish reliable PSP linkages. However, the framework is highly extensible for including more details of the microstructure (additional local states as well as higherorder spatial correlations) as more data becomes available.

\section{Acknowledgments}

The authors wish to acknowledge funding from NIST 70NANB14H191.

\section{References}

[1] Materials Genome Initiative for Global Competitiveness., in: N.S.a.T. Council (Ed.) 2011.

[2] T.M. Pollock, J.E. Allison, D.G. Backman, M.C. Boyce, M. Gersh, E.A. Holm, R. LeSar, M. Long, A.C.P. IV, J.J. Schirra, D.D. Whitis, C. Woodward, Integrated Computational Materials Engineering: A Transformational Discipline for Improved Competitiveness and National Security, The National Acamedies Press, Washington DC, 2008.

[3] A National Strategic Plan for Advanced Manufacturing, in: N.S.a.T.C. Executive Office of the President (Ed.) Feb 2012.

[4] M. Drosback, Materials Genome Initiative: Advances and Initiatives, JOM 66(3) (2014).

[5] G.J. Schmitz, U. Prahl, ICMEg-the Integrated Computational Materials Engineering expert group-a new European coordination action, Integrating Materials and Manufacturing Innovation 3(1) (2014) 2.

[6] D. Jarvis, Metallurgy Europe: A Renaissance Programme for 2012-2022, Adv. Phys. 61 (2012) 665-743. 
[7] W.F. Maier, K. Stowe, S. Sieg, Combinatorial and high-throughput materials science, Angew. Chem.-Int. Edit. 46(32) (2007) 6016-6067.

[8] R. Potyrailo, K. Rajan, K. Stoewe, I. Takeuchi, B. Chisholm, H. Lam, Combinatorial and High-Throughput Screening of Materials Libraries: Review of State of the Art, ACS Comb. Sci. 13(6) (2011) 579-633.

[9] C.G. Simon, S. Lin-Gibson, Combinatorial and High-Throughput Screening of Biomaterials, Adv. Mater. 23(3) (2011) 369-387.

[10] J.C. Zhao, High-throughput experimental tools for the materials genome initiative, Chin. Sci. Bull. 59(15) (2014) 1652-1661.

[11] M.L. Green, I. Takeuchi, J.R. Hattrick-Simpers, Applications of high throughput (combinatorial) methodologies to electronic, magnetic, optical, and energy-related materials, J. Appl. Phys. 113(23) (2013).

[12] H. Springer, D. Raabe, Rapid alloy prototyping: Compositional and thermomechanical high throughput bulk combinatorial design of structural materials based on the example of 30Mn-1.2C-xAl triplex steels, Acta Materialia 60(12) (2012) 4950-4959.

[13] F. Warchomicka, C. Poletti, M. Stockinger, T. Henke, Microstructure Evolution During Hot Deformation of Ti-6Al-4v Double Cone Specimens, International Journal of Material Forming 3 (2010) 215-218.

[14] D.B. Miracle, J.D. Miller, O.N. Senkov, C. Woodward, M.D. Uchic, J. Tiley, Exploration and Development of High Entropy Alloys for Structural Applications, Entropy 16(1) (2014) 494-525.

[15] J.C. Zhao, M.R. Jackson, L.A. Peluso, L.N. Brewer, A diffusion-multiple approach for mapping phase diagrams, hardness, and elastic modulus, Jom-Journal of the Minerals Metals \& Materials Society 54(7) (2002) 42-45.

[16] J.C. Zhao, A combinatorial approach for efficient mapping of phase diagrams and properties, J Mater Res 16(6) (2001) 1565-1578.

[17] O.L. Warren, T.J. Wyrobek, Nanomechanical property screening of combinatorial thin-film libraries by nanoindentation, Measurement Science \& Technology 16(1) (2005) 100-110.

[18] V.V. Shastry, V.D. Divya, M.A. Azeem, A. Paul, D. Dye, U. Ramamurty, Combining indentation and diffusion couple techniques for combinatorial discovery of high temperature shape memory alloys, Acta Materialia 61(15) (2013) 5735-5742. [19] S.M. Han, R. Shah, R. Banerjee, G.B. Viswanathan, B.M. Clemens, W.D. Nix, Combinatorial studies of mechanical properties of $\mathrm{Ti}-\mathrm{Al}$ thin films using nanoindentation, Acta Materialia 53(7) (2005) 2059-2067.

[20] E. Menendez, C. Templier, G. Abrasonis, J.F. Lopez-Barbera, J. Nogues, K. Temst, J. Sort, A combinatorial study of the mechanical and magnetic properties of a gradually nitrided austenitic stainless steel single crystal, Crystengcomm 16(17) (2014) 3515-3520. 
[21] C.A. Tweedie, D.G. Anderson, R. Langer, K.J. Van Vliet, Combinatorial material mechanics: High-throughput polymer synthesis and nanomechanical screening, Adv. Mater. 17(21) (2005) 2599-+.

[22] D.L. McDowell, G.B. Olson, Concurrent Design of Hierarchical Materials and Structures, Scientific Modeling and Simulation 15 (2008) 207-240.

[23] C.E. Campbell, G.B. Olson, Systems design of high performance stainless steels

I. Conceptual and computational design, Journal of Computer-Aided Materials Design 7 (2001) 145-170.

[24] C.E. Campbell, G.B. Olson, Systems design of high performance stainless steels II. Prototype characterization, Journal of Computer-Aided Materials Design 7 (2001) 171-194.

[25] G.B. Olson, Pathways of Discovery Designing a new material world, Science 228(12) (2000) 933-998.

[26] G.B. Olson, Computational Design of Hierarchically Structured Materials, Science 277(29) (1997) 1237-1242.

[27] G.B. Olson, Systems design of hierarchically structured materials: Advanced steels, Journal of Computer-Aided Materials Design 4 (1997) 143-156.

[28] J.H. Panchal, S.R. Kalidindi, D.L. McDowell, Key computational modeling issues in integrated computational materials engineering, Computer-Aided Design 45(1) (2013) 4-25.

[29] D.L. McDowell, J.H. Panchal, H.-J. Choi, C.C. Seepersad, J.K. Allen, F. Mistree, Integrated Design of Multiscale, Multifunctional Materials and Products, Elsevier2009.

[30] B.L. Adams, S.R. Kalidindi, D. Fullwood, Microstructure Sensitive Design for Performance Optimization, Butterworth-Heinemann2012.

[31] D.T. Fullwood, S.R. Niezgoda, B.L. Adams, S.R. Kalidindi, Microstructure sensitive design for performance optimization, Progress in Materials Science 55(6) (2010) 477-562.

[32] D.L. McDowell, S.R. Kalidindi, The materials innovation ecosystem: A key enabler for the Materials Genome Initiative, MRS Bulletin 41(04) (2016) 326-337. [33] S.R. Kalidindi, Data science and cyberinfrastructure: critical enablers for accelerated development of hierarchical materials, International Materials Reviews 60(3) (2015) 150-168.

[34] S.R. Kalidindi, Hierarchical Materials Informatics, Butterworth Heinemann2015.

[35] Standard Test Methods and Definitions for Mechanical Testing of Steel Products, ASTM International, 2016.

[36] H.K. Zeytin, C. Kubilay, H. Aydin, Investigation of dual phase transformation of commercial low alloy steels: Effect of holding time at low inter-critical annealing temperatures, Materials Letters 62(17-18) (2008) 2651-2653. 
[37] V.H. Baltazar Hernandez, S.K. Panda, M.L. Kuntz, Y. Zhou, Nanoindentation and microstructure analysis of resistance spot welded dual phase steel, Materials Letters 64(2) (2010) 207-210.

[38] X. Xu, S. van der Zwaag, W. Xu, The effect of ferrite-martensite morphology on the scratch and abrasive wear behaviour of a dual phase construction steel, Wear 348-349 (2016) 148-157.

[39] L. Zhang, T. Ohmura, A. Shibata, K. Tsuzaki, Characterization of local deformation behavior of $\mathrm{Fe}-\mathrm{Ni}$ lenticular martensite by nanoindentation, Materials Science and Engineering: A 527(7-8) (2010) 1869-1874.

[40] R.D.K. Misra, P. Venkatsurya, K.M. Wu, L.P. Karjalainen, Ultrahigh strength martensite-austenite dual-phase steels with ultrafine structure: The response to indentation experiments, Materials Science and Engineering: A 560 (2013) 693-699. [41] S.S. Ghasemi Banadkouki, E. Fereiduni, Effect of prior austenite carbon partitioning on martensite hardening variation in a low alloy ferrite-martensite dual phase steel, Materials Science and Engineering: A 619 (2014) 129-136.

[42] Y. Mazaheri, A. Kermanpur, A. Najafizadeh, Nanoindentation study of ferritemartensite dual phase steels developed by a new thermomechanical processing, Materials Science and Engineering: A 639 (2015) 8-14.

[43] G. Cheng, K.S. Choi, X. Hu, X. Sun, Determining individual phase properties in a multi-phase Q\&amp;P steel using multi-scale indentation tests, Materials Science and Engineering: A 652 (2016) 384-395.

[44] F.K. Yan, B.B. Zhang, H.T. Wang, N.R. Tao, K. Lu, Nanoindentation characterization of nano-twinned grains in an austenitic stainless steel, Scripta Materialia 112 (2016) 19-22.

[45] V.L. de la Concepción, H.N. Lorusso, H.G. Svoboda, Effect of Carbon Content on Microstructure and Mechanical Properties of Dual Phase Steels, Procedia Materials Science 8 (2015) 1047-1056.

[46] T. Ohmura, K. Tsuzaki, S. Matsuoka, Evaluation of the matrix strength of Fe$0.4 \mathrm{wt} \% \mathrm{C}$ tempered martensite using nanoindentation techniques, Philosophical Magazine A 82(10) (2002) 1903-1910.

[47] C.C. Tasan, M. Diehl, D. Yan, M. Bechtold, F. Roters, L. Schemmann, C. Zheng, N. Peranio, D. Ponge, M. Koyama, K. Tsuzaki, D. Raabe, An Overview of Dual-Phase Steels: Advances in Microstructure-Oriented Processing and Micromechanically Guided Design, Annual Review of Materials Research 45(1) (2015) 391-431.

[48] K. Mukherjee, S.S. Hazra, M. Militzer, Grain Refinement in Dual-Phase Steels, Metallurgical and Materials Transactions A 40(9) (2009) 2145-2159.

[49] L.E. Samuels, Tempering of Martensite, Metallography, Microstructure, and Analysis 3(1) (2014) 70-90. 
[50] W.C. Jeong, Relationship between mechanical properties and microstructure in a $1.5 \% \mathrm{Mn}-0.3 \%$ Mo ultra-low carbon steel with bake hardening, Materials Letters 61(11-12) (2007) 2579-2583.

[51] P. Tsipouridis, Mechanical Properties of Dual Phase Steels, Hieronymus2006. [52] L. Schemmann, S. Zaefferer, D. Raabe, F. Friedel, D. Mattissen, Alloying effects on microstructure formation of dual phase steels, Acta Materialia 95 (2015) 386-398.

[53] S. Pathak, S.R. Kalidindi, Spherical nanoindentation stress-strain curves, Materials Science and Engineering: R: Reports 91 (2015) 1-36.

[54] S.R. Kalidindi, S. Pathak, Determination of the effective zero-point and the extraction of spherical nanoindentation stress-strain curves, Acta Materialia 56(14) (2008) 3523-3532.

[55] S.R. Kalidindi, S.J. Vachhani, Mechanical characterization of grain boundaries using nanoindentation, Current Opinion in Solid State and Materials Science (2014). [56] S. Pathak, S.R. Kalidindi, N.A. Mara, Investigations of orientation and length scale effects on micromechanical responses in polycrystalline zirconium using spherical nanoindentation, Scripta Materialia 113 (2016) 241-245.

[57] S. Pathak, J. Shaffer, S.R. Kalidindi, Determination of an effective zero-point and extraction of indentation stress-strain curves without the continuous stiffness measurement signal, Scripta Materialia 60(6) (2009) 439-442.

[58] S. Pathak, D. Stojakovic, S.R. Kalidindi, Measurement of the local mechanical properties in polycrystalline samples using spherical nanoindentation and orientation imaging microscopy, Acta Materialia 57(10) (2009) 3020-3028.

[59] S.J. Vachhani, R.D. Doherty, S.R. Kalidindi, Studies of grain boundary regions in deformed polycrystalline aluminum using spherical nanoindentation, International Journal of Plasticity 81 (2016) 87-101.

[60] S.J. Vachhani, S.R. Kalidindi, Grain-scale measurement of slip resistances in aluminum polycrystals using spherical nanoindentation, Acta Materialia 90 (2015) 27-36.

[61] J.S. Weaver, A. Khosravani, A. Castillo, S.R. Kalidindi, High throughput exploration of process-property linkages in Al-6061 using instrumented spherical microindentation and microstructurally graded samples, Integrating Materials and Manufacturing Innovation 5(1) (2016) 1-20.

[62] S.Y.P. Allain, O. Bouaziz, I. Pushkareva, C.P. Scott, Towards the microstructure design of DP steels: A generic size-sensitive mean-field mechanical model, Materials Science and Engineering: A 637 (2015) 222-234.

[63] S.K. Paul, N. Stanford, T. Hilditch, Effect of martensite volume fraction on low cycle fatigue behaviour of dual phase steels: Experimental and microstructural investigation, Materials Science and Engineering: A 638 (2015) 296-304. 
[64] B. Demir, M. Erdoğan, The hardenability of austenite with different alloy content and dispersion in dual-phase steels, Journal of Materials Processing Technology 208(1-3) (2008) 75-84.

[65] N.K. P.D. Sudersanan, S. Aprameyan and Dr.U.N. Kempaiah, The Effect of Carbon Content in Martensite on the Strength of Dual Phase Steel

Bonfring International Journal of Industrial Engineering and Management Science 2(2) (2012) 01-04.

[66] M. Asadi, B.C. De Cooman, H. Palkowski, Influence of martensite volume fraction and cooling rate on the properties of thermomechanically processed dual phase steel, Materials Science and Engineering: A 538 (2012) 42-52.

[67] H. Seyedrezai, Thermo-Mechanical Processing of Dual-Phase Steels and Its Effects on the Work Hardening Behaviour, Mechanical and Materials Engineering, Queen's University, 2014.

[68] A. Gupta, A. Cecen, S. Goyal, A.K. Singh, S.R. Kalidindi, Structure-property linkages using a data science approach: Application to a non-metallic inclusion/steel composite system, Acta Materialia 91 (2015) 239-254.

[69] S.R. Kalidindi, Hierarchical Materials Informatics: Novel Analytics for Materials Data, Elsevier2015.

[70] S. Torquato, Random Heterogeneous Materials, Springer-Verlag, New York, 2002.

[71] S.R. Niezgoda, A.K. Kanjarla, S.R. Kalidindi, Novel microstructure quantification framework for databasing, visualization, and analysis of microstructure data, Integrating Materials and Manufacturing Innovation 2:3 (2013). [72] S.R. Niezgoda, D.T. Fullwood, S.R. Kalidindi, Delineation of the space of 2point correlations in a composite material system, Acta Materialia 56(18) (2008) 5285-5292.

[73] B.L. Adams, G. Xiang, S.R. Kalidindi, Finite approximations to the secondorder properties closure in single phase polycrystals, Acta Materialia 53(13) (2005) 3563-3577.

[74] S.R. Niezgoda, Y.C. Yabansu, S.R. Kalidindi, Understanding and visualizing microstructure and microstructure variance as a stochastic process, Acta Materialia 59(16) (2011) 6387-6400.

[75] S.R. Kalidindi, S.R. Niezgoda, A.A. Salem, Microstructure informatics using higher-order statistics and efficient data-mining protocols, JOM 63(4) (2011) 34-41. [76] X. Gao, C.P. Przybyla, B.L. Adams, Methodology for Recovering and Analyzing Two-Point Pair Correlation Functions in Polycrystalline Materials, Metallurgical and Materials Transactions A 37(8) (2006) 2379-2387.

[77] T.A. Mason, B.L. Adams, Use of microstructural statistics in predicting polycrystalline material properties, Metallurgical and Materials Transactions A: Physical Metallurgy and Materials Science 30(4) (1999) 969-979. 
[78] S.M. Qidwai, D.M. Turner, S.R. Niezgoda, A.C. Lewis, A.B. Geltmacher, D.J. Rowenhorst, S.R. Kalidindi, Estimating response of polycrystalline materials using sets of weighted statistical volume elements (WSVEs), Acta Materialia 60 (2012) 5284-5299.

[79] T. Waterschoot, K. Verbeken, B.C. De Cooman, Tempering Kinetics of the Martensitic Phase in DP Steel, ISIJ International 46(1) (2006) 138-146.

[80] A. Hüseyin, K.Z. Havva, K. Ceylan, Effect of Intercritical Annealing Parameters on Dual Phase Behavior of Commercial Low-Alloyed Steels, Journal of Iron and Steel Research, International 17(4) (2010) 73-78.

[81] T. Hilditch, H. Beladi, P. Hodgson, N. Stanford, Role of microstructure in the low cycle fatigue of multi-phase steels, Materials Science and Engineering: A 534 (2012) 288-296.

[82] A. Ramazani, S. Bruehl, T. Gerber, W. Bleck, U. Prahl, Quantification of bake hardening effect in DP600 and TRIP700 steels, Materials \& Design 57 (2014) 479486.

[83] C.F. Kuang, J. Li, S.G. Zhang, J. Wang, H.F. Liu, A.A. Volinsky, Effects of quenching and tempering on the microstructure and bake hardening behavior of ferrite and dual phase steels, Materials Science and Engineering: A 613 (2014) 178183.

[84] C.-s. Li, Z.-x. Li, Y.-m. Cen, B. Ma, G. Huo, Microstructure and mechanical properties of dual phase strip steel in the overaging process of continuous annealing, Materials Science and Engineering: A 627 (2015) 281-289.

[85] I.B. Timokhina, P.D. Hodgson, E.V. Pereloma, Transmission Electron Microscopy Characterization of the Bake-Hardening Behavior of TransformationInduced Plasticity and Dual-Phase Steels, Metallurgical and Materials Transactions A 38(10) (2007) 2442-2454.

[86] K. LINDQVIST, Bake hardening effect in advanced high-strength steels, Department of Applied Mechanics, CHALMERS UNIVERSITY OF TECHNOLOGY, 2013.

[87] Y. Cao, J. Ahlström, B. Karlsson, The influence of temperatures and strain rates on the mechanical behavior of dual phase steel in different conditions, Journal of Materials Research and Technology 4(1) (2015) 68-74.

[88] M. Šebek, P. Horňak, P. Zimovčák, Effect of Annealing on the Microstructure Evolution and Mechanical Properties of Dual Phase Steel, Materials Science Forum 782 (2014) 111-116.

[89] L.T. Robertson, T.B. Hilditch, P.D. Hodgson, The effect of prestrain and bake hardening on the low-cycle fatigue properties of TRIP steel, International Journal of Fatigue 30(4) (2008) 587-594. 
[90] C.-f. Kuang, S.-g. Zhang, J. Li, J. Wang, P. Li, Effect of temper rolling on the bake-hardening behavior of low carbon steel, International Journal of Minerals, Metallurgy, and Materials 22(1) (2015) 32-36.

[91] S. Berbenni, V. Favier, X. Lemoine, M. Berveiller, A micromechanical approach to model the bake hardening effect for low carbon steels, Scripta Materialia 51(4) (2004) 303-308.

[92] C.-f. Kuang, J. Wang, J. Li, S.-g. Zhang, H.-f. Liu, H.-1. Yang, Effect of Continuous Annealing on Microstructure and Bake Hardening Behavior of Low Carbon Steel, Journal of Iron and Steel Research, International 22(2) (2015) 163170.

[93] H. Wang, W. Shi, Y.-1. He, X.-g. Lu, L. Li, Effect of Overaging on Solute Distributions and Bake Hardening Phenomenon in Bake Hardening Steels, Journal of Iron and Steel Research, International 19(1) (2012) 53-59.

[94] L. Durrenberger, X. Lemoine, A. Molinari, Effects of pre-strain and bakehardening on the crash properties of a top-hat section, Journal of Materials Processing Technology 211(12) (2011) 1937-1947.

[95] S. Kilic, F. Ozturk, T. Sigirtmac, G. Tekin, Effects of Pre-strain and Temperature on Bake Hardening of TWIP900CR Steel, Journal of Iron and Steel Research, International 22(4) (2015) 361-365.

[96] A.A. Vasilyev, H.-C. Lee, N.L. Kuzmin, Nature of strain aging stages in bake hardening steel for automotive application, Materials Science and Engineering: A 485(1-2) (2008) 282-289.

[97] I.B. Timokhina, E.V. Pereloma, S.P. Ringer, R.K. Zheng, P.D. Hodgson, Characterization of the Bake-hardening Behavior of Transformation Induced Plasticity and Dual-phase Steels Using Advanced Analytical Techniques, ISIJ International 50(4) (2010) 574-582.

[98] D.A. Korzekwa, D.K. Matlock, G. Krauss, Dislocation substructure as a function of strain in a dual-phase steel, Metallurgical Transactions A 15(6) (1984) 1221-1228.

[99] M. Calcagnotto, D. Ponge, E. Demir, D. Raabe, Orientation gradients and geometrically necessary dislocations in ultrafine grained dual-phase steels studied by 2D and 3D EBSD, Materials Science and Engineering: A 527(10-11) (2010) 2738-2746.

[100] E.V. Nesterova, S. Bouvier, B. Bacroix, Microstructure evolution and mechanical behavior of a high strength dual-phase steel under monotonic loading, Materials Characterization 100 (2015) 152-162.

[101] A.H. Cottrell, B.A. Bilby, Dislocation Theory of Yielding and Strain Ageing of Iron, Proceedings of the Physical Society. Section A 62(1) (1949) 49.

[102] S. Ghanei, A. Saheb Alam, M. Kashefi, M. Mazinani, Nondestructive characterization of microstructure and mechanical properties of intercritically 
annealed dual-phase steel by magnetic Barkhausen noise technique, Materials Science and Engineering: A 607 (2014) 253-260.

[103] N. Shukla, S. Das, S. Maji, S.R. Chowdhury, B.K. Show, Effect of Preintercritical Annealing Treatments on the Microstructure and Mechanical Properties of $0.33 \%$ Carbon Dual-Phase Steel, Journal of Materials Engineering and Performance 24(12) (2015) 4958-4965.

[104] C.-f. Kuang, S.-g. Zhang, J. Li, J. Wang, H.-f. Liu, Effects of pre-strain and baking parameters on the microstructure and bake-hardening behavior of dual-phase steel, International Journal of Minerals, Metallurgy, and Materials 21(8) (2014) 766771.

[105] M. Asadi, Influence of the Hot Rolling Process on the Mechanical Behavior of Dual Phase Steels, Fakultät für Natur- und Materialwissenschaftlichen, Technischen Universität Clausthal, 2010.

[106] V. Colla, M. Sanctis, A. Dimatteo, G. Lovicu, A. Solina, R. Valentini, Strain Hardening Behavior of Dual-Phase Steels, Metallurgical and Materials Transactions A 40(11) (2009) 2557-2567.

[107] S.R. Kalidindi, S.J. Vachhani, Mechanical characterization of grain boundaries using nanoindentation, Current Opinion in Solid State and Materials Science 18(4) (2014) 196-204.

[108] J.S. Weaver, A. Khosravani, A. Castillo, S.R. Kalidindi, High Throughput Exploration of Process-Property Linkages in Al-6061 using Instrumented Spherical Microindentation and Microstructurally Graded Samples, Integrating Materials and Manufacturing Innovation In press (2016).

[109] S.R. Kalidindi, S. Pathak, Determination of the effective zero-point and the extraction of spherical nanoindentation stress-strain curves, Acta Materialia 56(14) (2008) 3523-3532.

[110] M. Koopman, Z.Z. Fang, X. Wang, P.K. Mehrotra, Properties and Selection of Cemented Carbides, Powder Metallurgy, ASM International, ASM Handbook, 2015, pp. 705-710.

[111] Overview of materials for Low Alloy Steel, MatWeb, http://www.matweb.com/.

[112] C.C. Tasan, J.P.M. Hoefnagels, M. Diehl, D. Yan, F. Roters, D. Raabe, Strain localization and damage in dual phase steels investigated by coupled in-situ deformation experiments and crystal plasticity simulations, International Journal of Plasticity 63 (2014) 198-210.

[113] F. Zhang, A. Ruimi, P.C. Wo, D.P. Field, Morphology and distribution of martensite in dual phase (DP980) steel and its relation to the multiscale mechanical behavior, Materials Science and Engineering: A 659 (2016) 93-103. 
[114] B. Hutchinson, L. Ryde, E. Lindh, K. Tagashira, Texture in hot rolled austenite and resulting transformation products, Materials Science and Engineering: A 257(1) (1998) 9-17.

[115] T. Waterschoot, L. Kestens, B.C. De Cooman, Hot rolling texture development in CMnCrSi dual-phase steels, Metallurgical and Materials Transactions A 33(4) (2002) 1091-1102.

[116] S. Zaefferer, J. Ohlert, W. Bleck, A study of microstructure, transformation mechanisms and correlation between microstructure and mechanical properties of a low alloyed TRIP steel, Acta Materialia 52(9) (2004) 2765-2778.

[117] S. Wright, Quantification of recrystallized fraction from orientation imaging scans, National Research Council of Canada, Proceedings of the Twelfth International Conference on Textures of Materials(ICOTOM-12). 1999, pp. 104109.

[118] A. Agnoli, M. Bernacki, R. Logé, J.-M. Franchet, J. Laigo, N. Bozzolo, Understanding and modeling of gain boundary pinning in Inconel718, 2012.

[119] C. Lim, Length scale effect on the microstructural evolution of copper layers in a roll-bonded copper-niobium composite, in: A. Rollett (Ed.) ProQuest Dissertations Publishing, 2008.

[120] A. Kundu, D.P. Field, Influence of plastic deformation heterogeneity on development of geometrically necessary dislocation density in dual phase steel, Materials Science and Engineering: A 667 (2016) 435-443.

[121] J. Kadkhodapour, S. Schmauder, D. Raabe, S. Ziaei-Rad, U. Weber, M. Calcagnotto, Experimental and numerical study on geometrically necessary dislocations and non-homogeneous mechanical properties of the ferrite phase in dual phase steels, Acta Materialia 59(11) (2011) 4387-4394.

[122] K. Kimura, K. Ushioda, E. Ishimaru, A. Takahashi, Role of hard martensite phase prior to cold-rolling on microstructure evolution after annealing in ferritic stainless steel, Materials Science and Engineering: A 663 (2016) 86-97.

[123] A. Cecen, T. Fast, S.R. Kalidindi, Versatile algorithms for the computation of 2-point spatial correlations in quantifying material structure, Integrating Materials and Manufacturing Innovation 5(1) (2016) 1-15.

[124] D.T. Fullwood, S.R. Kalidindi, S.R. Niezgoda, A. Fast, N. Hampson, Gradient-based microstructure reconstructions from distributions using fast Fourier transforms, Materials Science and Engineering a-Structural Materials Properties Microstructure and Processing 494(1-2) (2008) 68-72.

[125] D.T. Fullwood, S.R. Niezgoda, S.R. Kalidindi, Microstructure reconstructions from 2-point statistics using phase-recovery algorithms, Acta Materialia 56(5) (2008) 942-948. 
[126] D.M. Turner, S.R. Kalidindi, Statistical construction of 3-D microstructures from 2-D exemplars collected on oblique sections, Acta Materialia 102 (2016) 136148.

[127] A. Choudhury, Y.C. Yabansu, S.R. Kalidindi, A. Dennstedt, Quantification and classification of microstructures in ternary eutectic alloys using 2-point spatial correlations and principal component analyses, Acta Materialia 110 (2016) 131-141. [128] T. Fast, O. Wodo, B. Ganapathysubramanian, S.R. Kalidindi, Microstructure taxonomy based on spatial correlations: Application to microstructure coarsening, Acta Materialia 108 (2016) 176-185.

[129] A. Agrawal, P.D. Deshpande, A. Cecen, G.P. Basavarsu, A.N. Choudhary, S.R. Kalidindi, Exploration of data science techniques to predict fatigue strength of steel from composition and processing parameters, Integrating Materials and Manufacturing Innovation 3(1) (2014) 1-19.

[130] A. Çeçen, T. Fast, E.C. Kumbur, S.R. Kalidindi, A data-driven approach to establishing microstructure-property relationships in porous transport layers of polymer electrolyte fuel cells, Journal of Power Sources 245 (2014) 144-153.

[131] S.R. Niezgoda, A.K. Kanjarla, S.R. Kalidindi, Novel microstructure quantification framework for databasing, visualization, and analysis of microstructure data, Integrating Materials and Manufacturing Innovation 2(1) (2013) 1-27.

[132] D.K. Patel, H.F. Al-Harbi, S.R. Kalidindi, Extracting single-crystal elastic constants from polycrystalline samples using spherical nanoindentation and orientation measurements, Acta Materialia 79 (2014) 108-116.

[133] J.S. Weaver, M.W. Priddy, D.L. McDowell, S.R. Kalidindi, On capturing the grain-scale elastic and plastic anisotropy of alpha-Ti with spherical nanoindentation and electron back-scattered diffraction, Acta Materialia 117 (2016) 23-34.

[134] J.S. Weaver, S.R. Kalidindi, Mechanical characterization of Ti-6Al-4V titanium alloy at multiple length scales using spherical indentation stress-strain measurements, Materials \& Design 111 (2016) 463-472.

[135] P. Steinmetz, Y.C. Yabansu, J. Hötzer, M. Jainta, B. Nestler, S.R. Kalidindi, Analytics for microstructure datasets produced by phase-field simulations, Acta Materialia 103 (2016) 192-203.

[136] S.R. Kalidindi, J.A. Gomberg, Z.T. Trautt, C.A. Becker, Application of data science tools to quantify and distinguish between structures and models in molecular dynamics datasets, Nanotechnology 26(34) (2015) 344006.

[137] A. CeCen, T. Fast, E.C. Kumbur, S.R. Kalidindi, A Data-driven Approach to Establishing Microstructure-Property Relationships in Porous Transport Layers of Polymer Electrolyte Fuel Cells, Journal of Power Sources 245 (2014) 144-153. 
[138] X. Dong, D.L. McDowell, S.R. Kalidindi, K.I. Jacob, Dependence of mechanical properties on crystal orientation of semi-crystalline polyethylene structures, Polymer 55(16) (2014) 4248-4257.

[139] M.T. David, R.N. Stephen, R.K. Surya, Efficient computation of the angularly resolved chord length distributions and lineal path functions in large microstructure datasets, Modelling and Simulation in Materials Science and Engineering 24(7) (2016) 075002.

[140] K.V. Mardia, J.T. Kent, J.M. Bibby, Multivariate analysis, (1980).

[141] I.K. Fodor, A survey of dimension reduction techniques, Technical Report UCRL-ID-148494, Lawrence Livermore National Laboratory, 2002.

[142] A. Hyvärinen, Survey on independent component analysis, (1999).

[143] J.R. Quinlan, Learning with continuous classes, 5th Australian joint conference on artificial intelligence, Singapore, 1992, pp. 343-348.

[144] M.A. Hearst, S.T. Dumais, E. Osman, J. Platt, B. Scholkopf, Support vector machines, IEEE Intelligent Systems and their Applications 13(4) (1998) 18-28.

[145] S.R. Kalidindi, A.J. Medford, D.L. McDowell, Vision for Data and Informatics in the Future Materials Innovation Ecosystem, JOM (2016) 1-12. [146] S.R. Kalidindi, M.D. Graef, Materials Data Science: Current Status and Future Outlook, Annual Review of Materials Research (2015). 


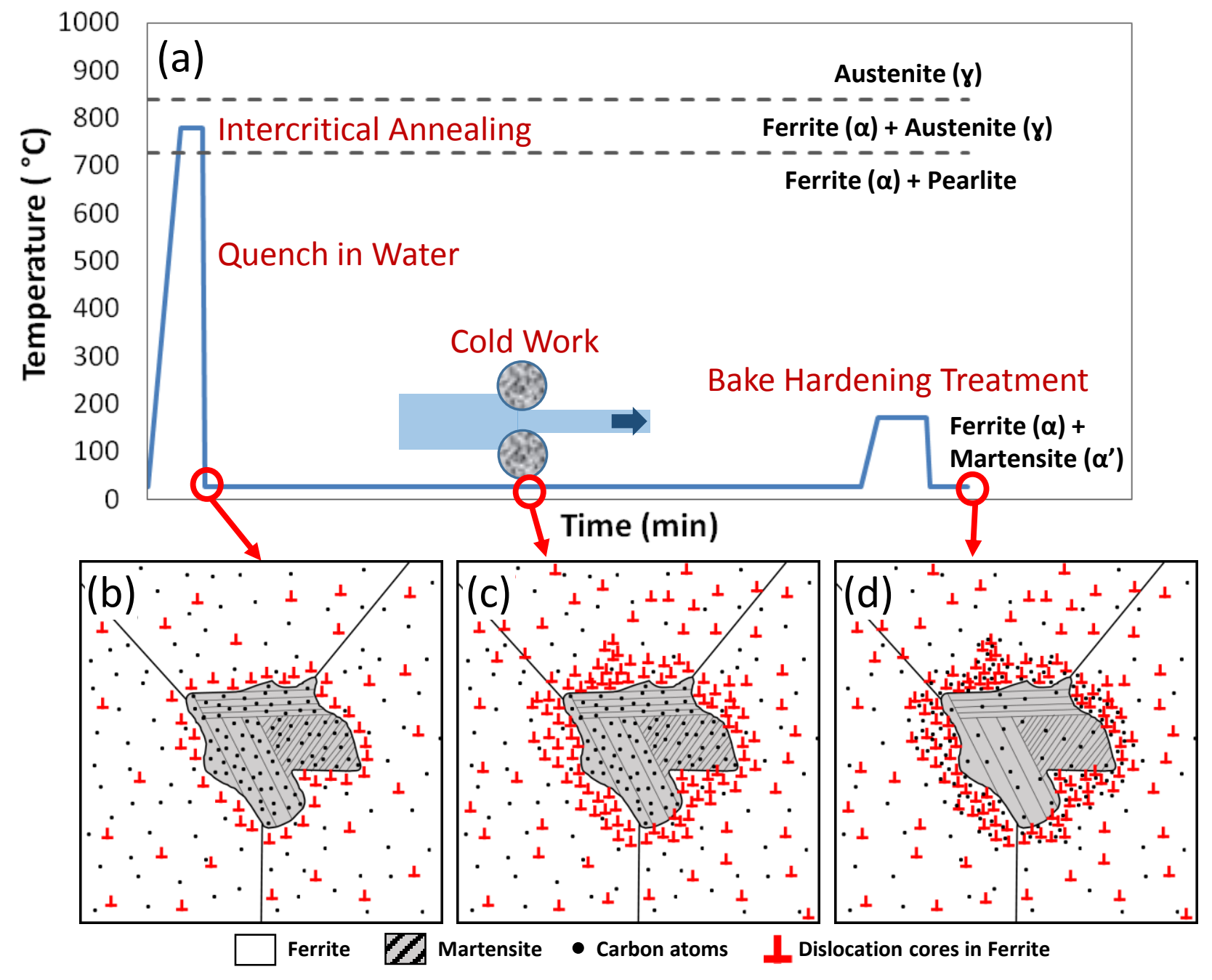

Figure 1. Schematic of a dual phase steel processing path and the expected strengthening mechanisms in each step of the thermo-mechanical process. 
Figure 2. Example microindentation load-displacement curve, and indentation stress-strain curve for a test on sample 750-10-170. The red data corresponds to the initial elastic segment used to determine the effective modulus.
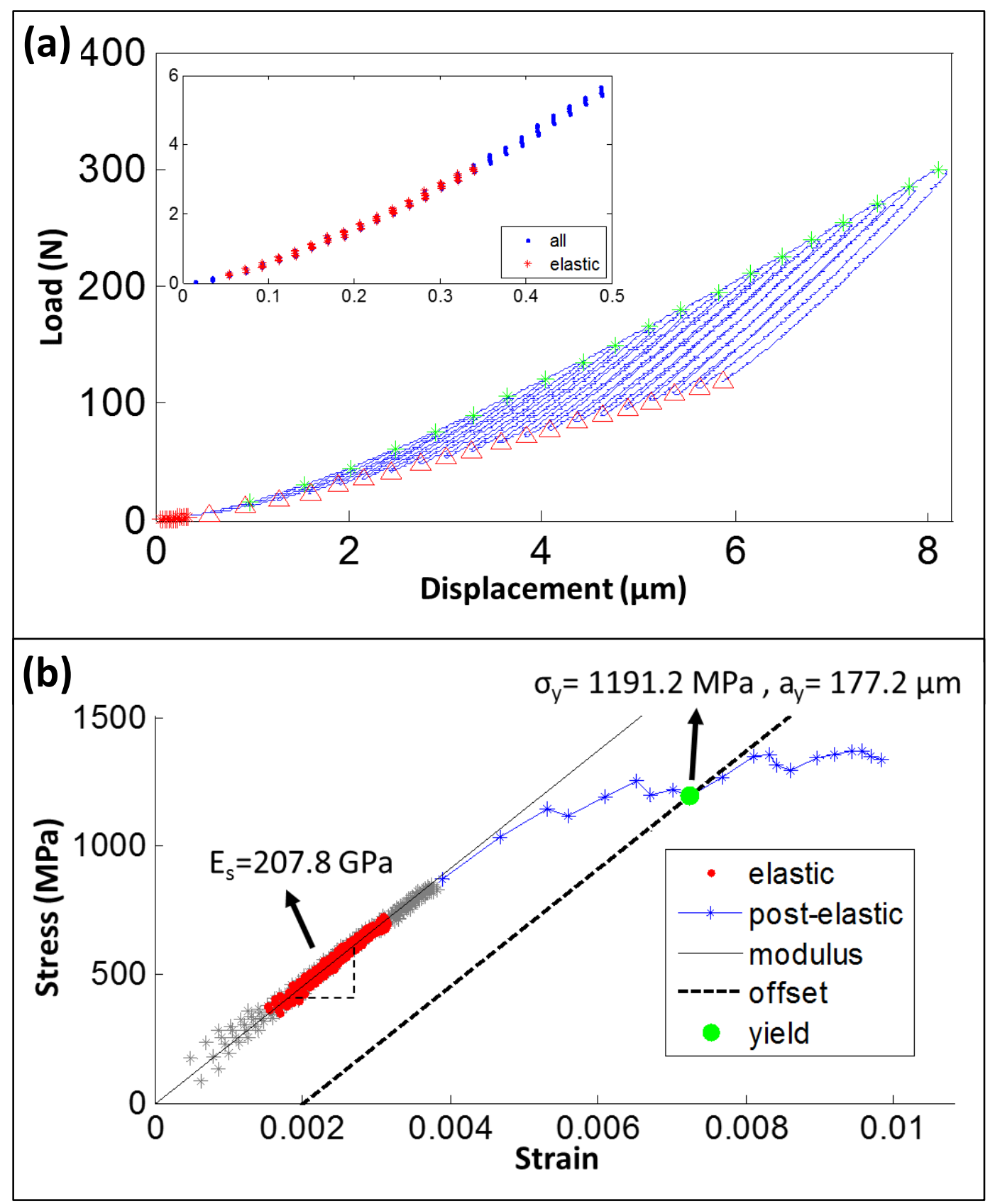

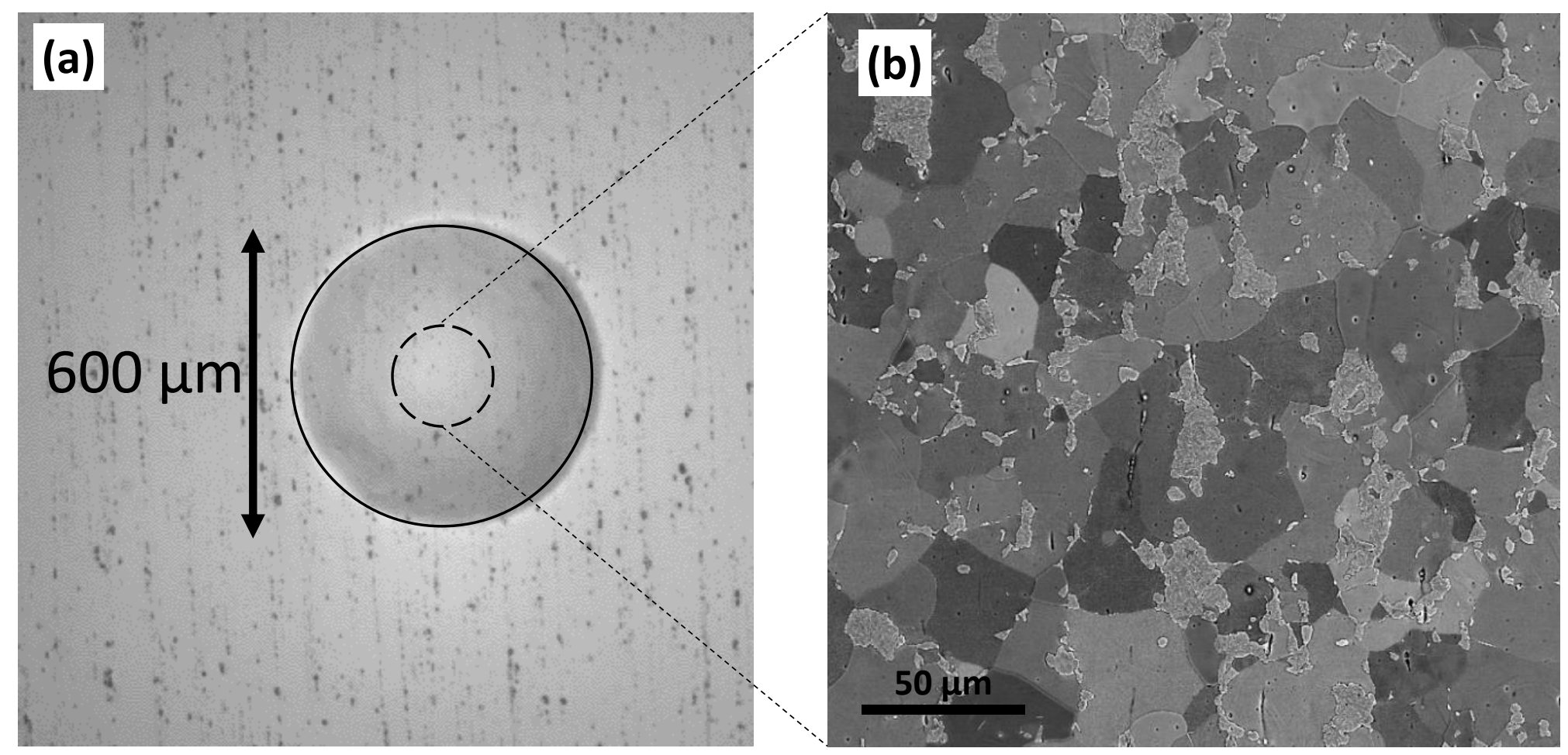

Figure 3. a) An optical micrograph showing the size of indentation after unloading, dashed line circle shows the estimated size of the contact area at yield. b) A representative BSE image with the same size as the area indicated by the dashed line. 


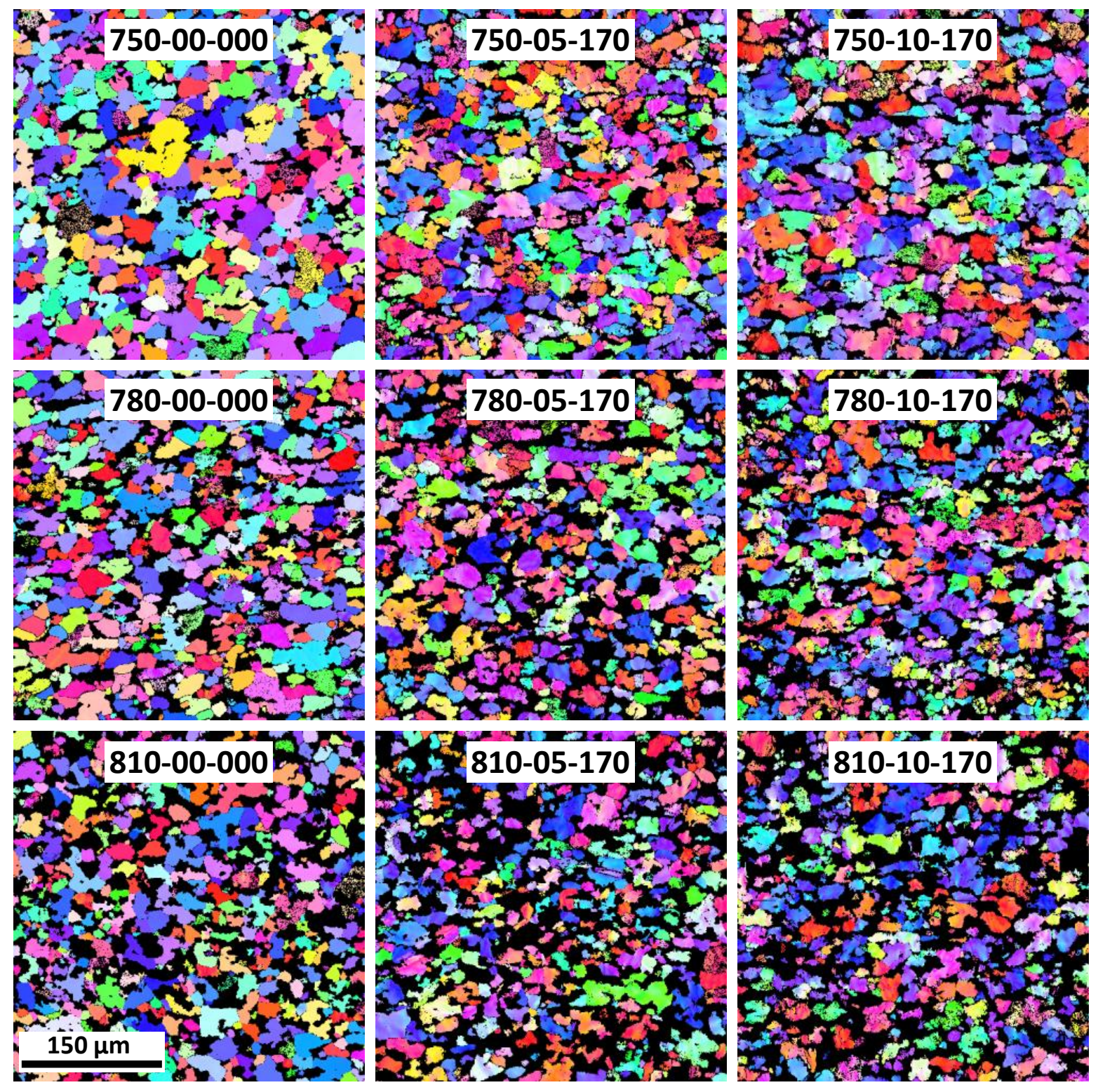

Figure 4. A sampling of microstructures produced in the present study. 

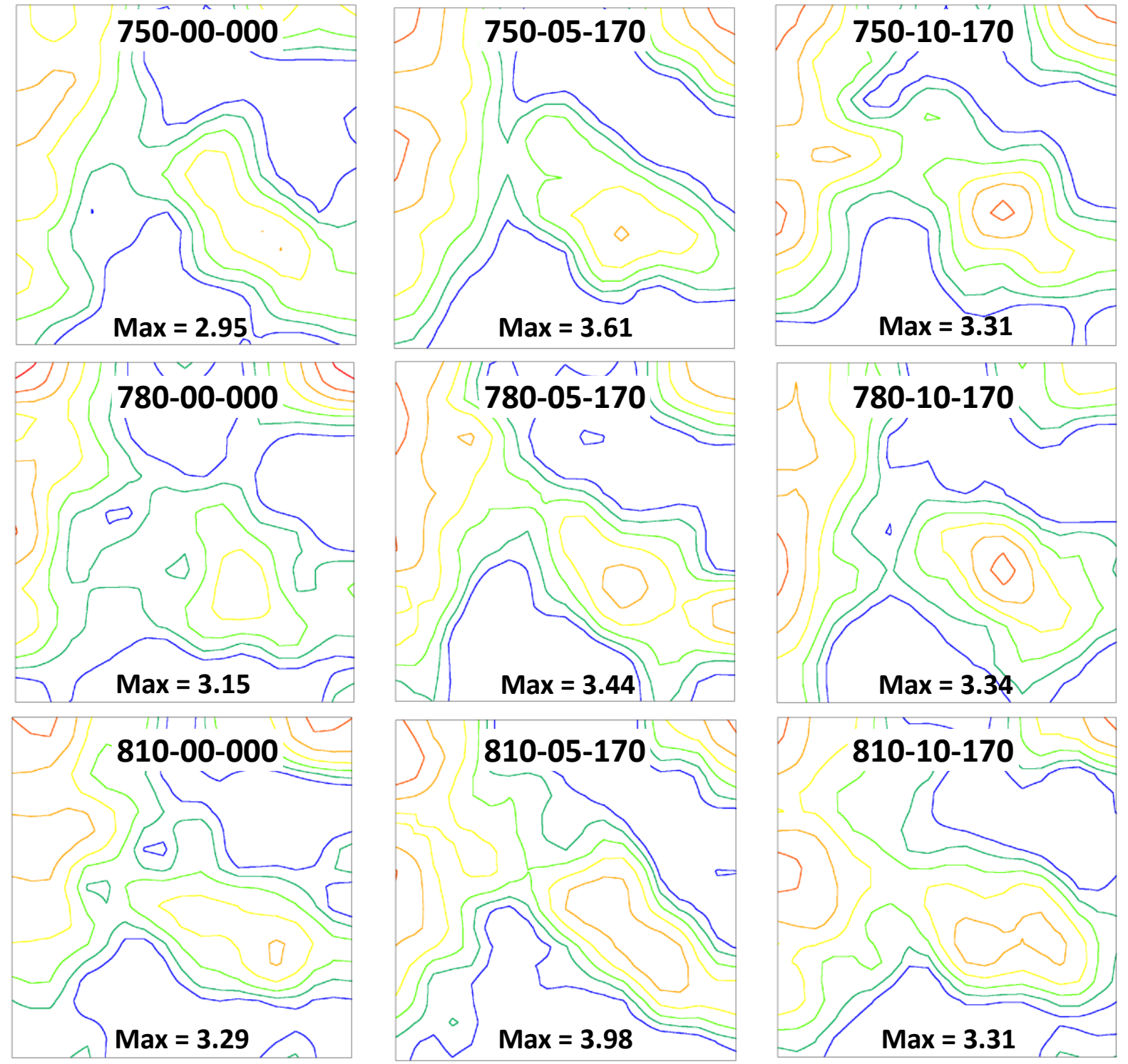

Figure 5. Ferrite textures in the $\varphi_{2}=45$ degree section of the ODF for the different samples produced in this study. 

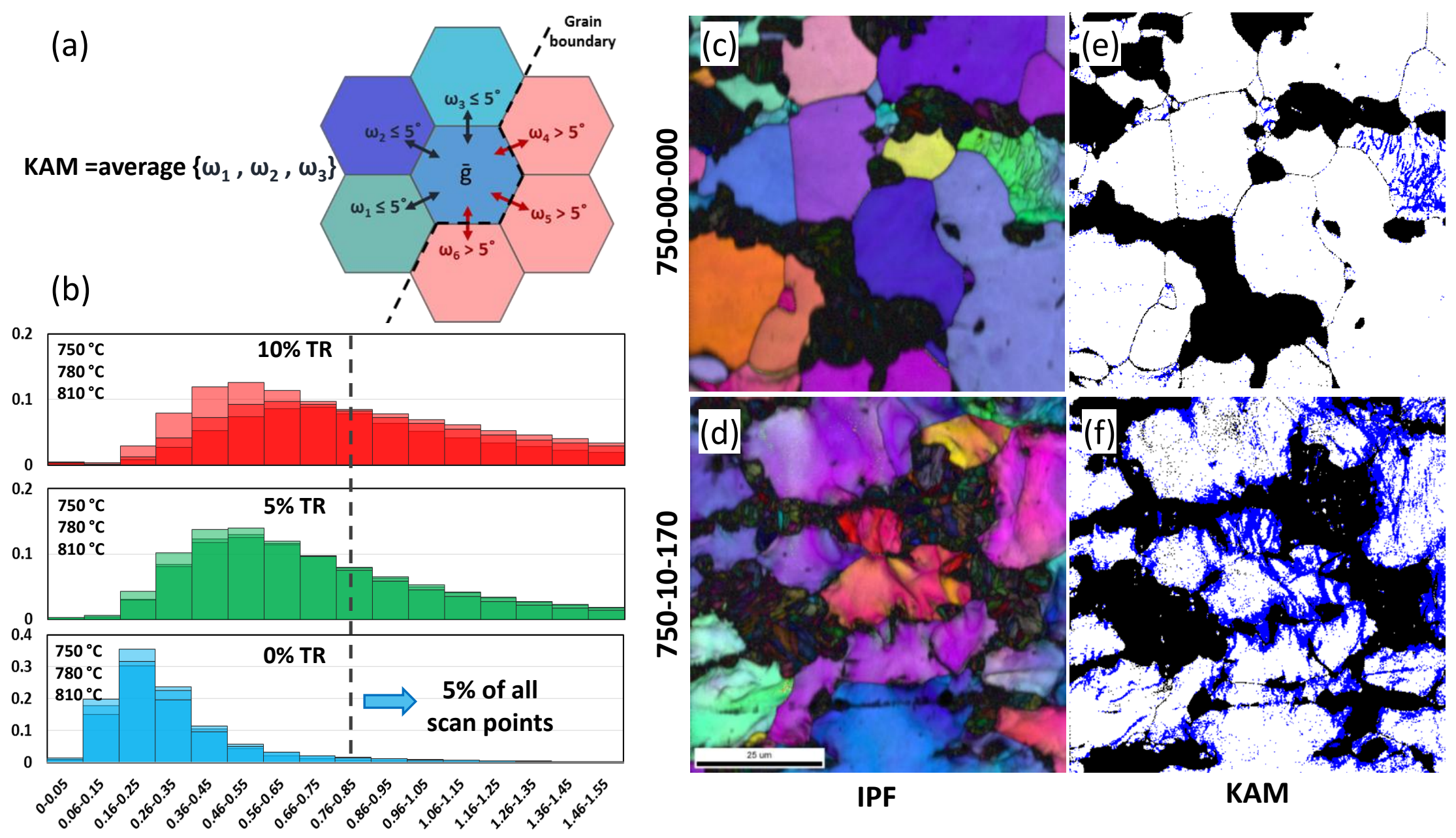

Figure 6. (a) Schematic of KAM definition for each pixel in the EBSD scan. (b) KAM distributions for the samples studied. (c) and (d) IPF maps from selected areas in samples with and without cold work. (e) and (f) Corresponding KAM maps identifying regions with KAM values larger than 0.8 in blue. 

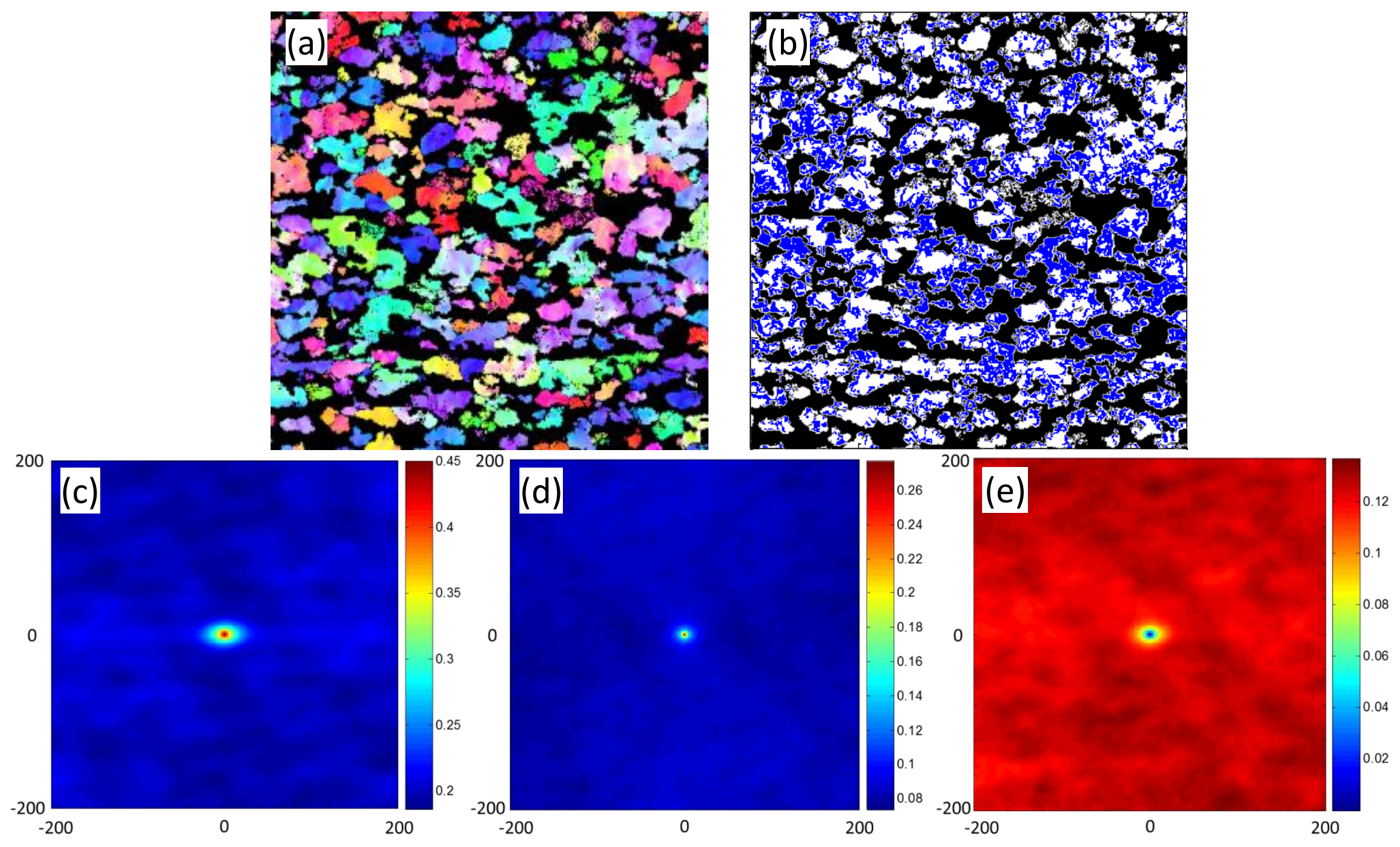

Figure 7. (a) An IPF map from the sample 780-10-170. (b) A map showing the three local states selected for the study - martensite as black pixels, deformed ferrite as blue pixels, and undeformed ferrite as white pixels. (c) Autocorrelation of martensite, (d) autocorrelation of deformed ferrite, and cross-correlation of martensite and deformed ferrite. 


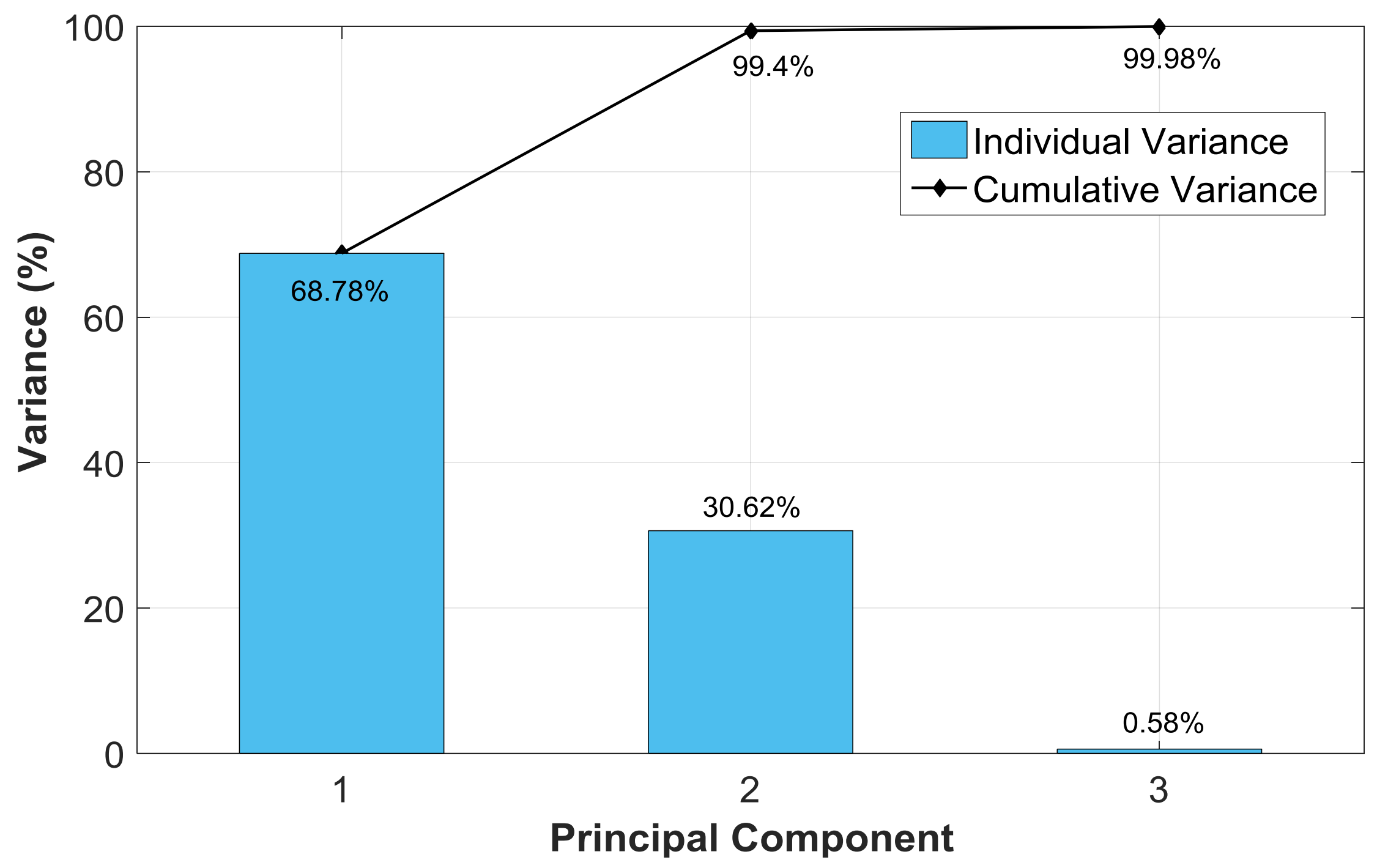

Figure 8. The individual and cumulative variances from the PCA for the first 3 principal components shows that PC1 and PC2 carry $99.4 \%$ of the total variance in the dataset. 


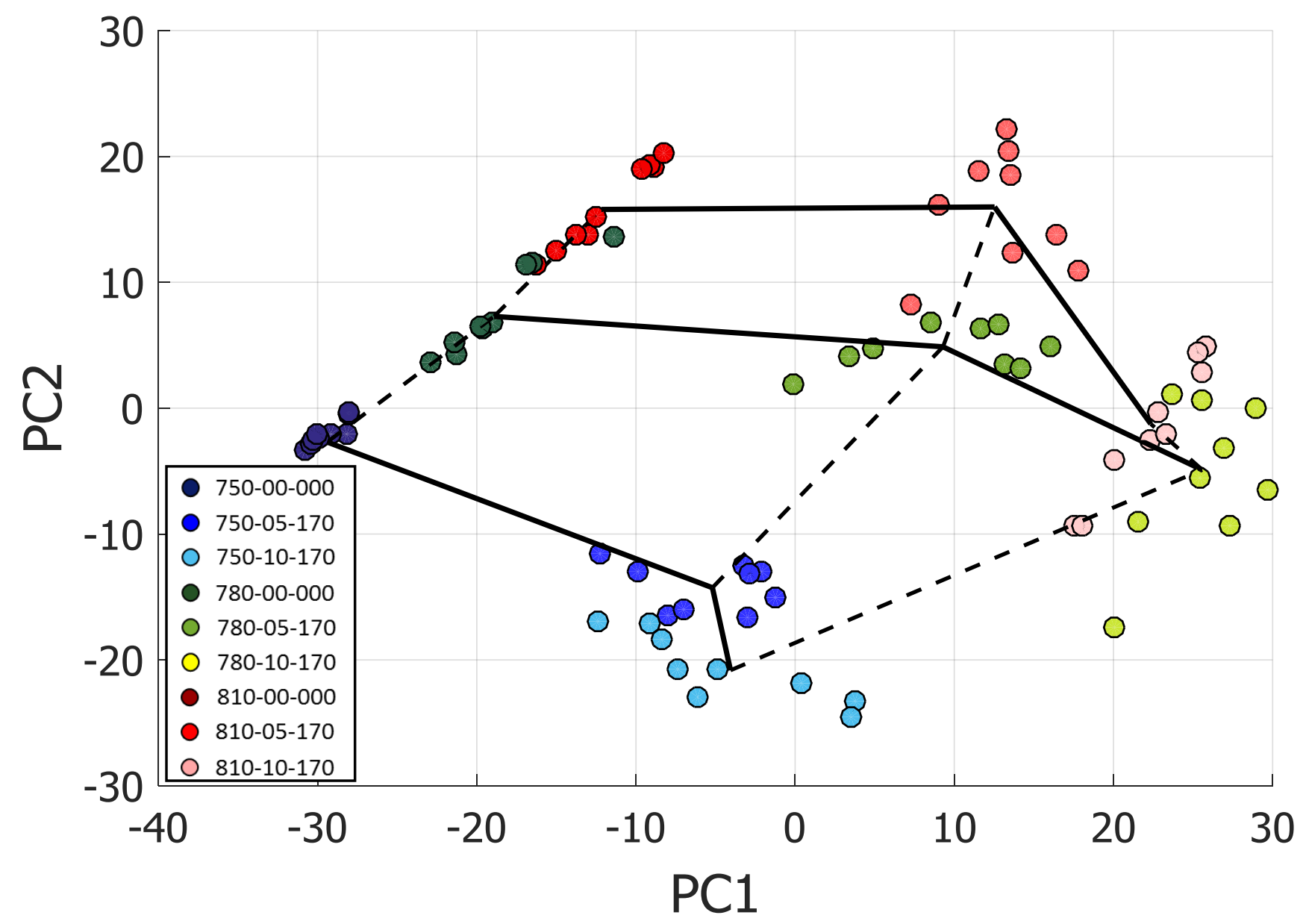

Figure 9. Low-dimensional representation of the entire ensemble of 81 micrographs obtained from PCA, colored by the different processing histories. Solid lines capture the influence of cold work, while the dashed lines show the effect of the intercritical annealing temperature. 

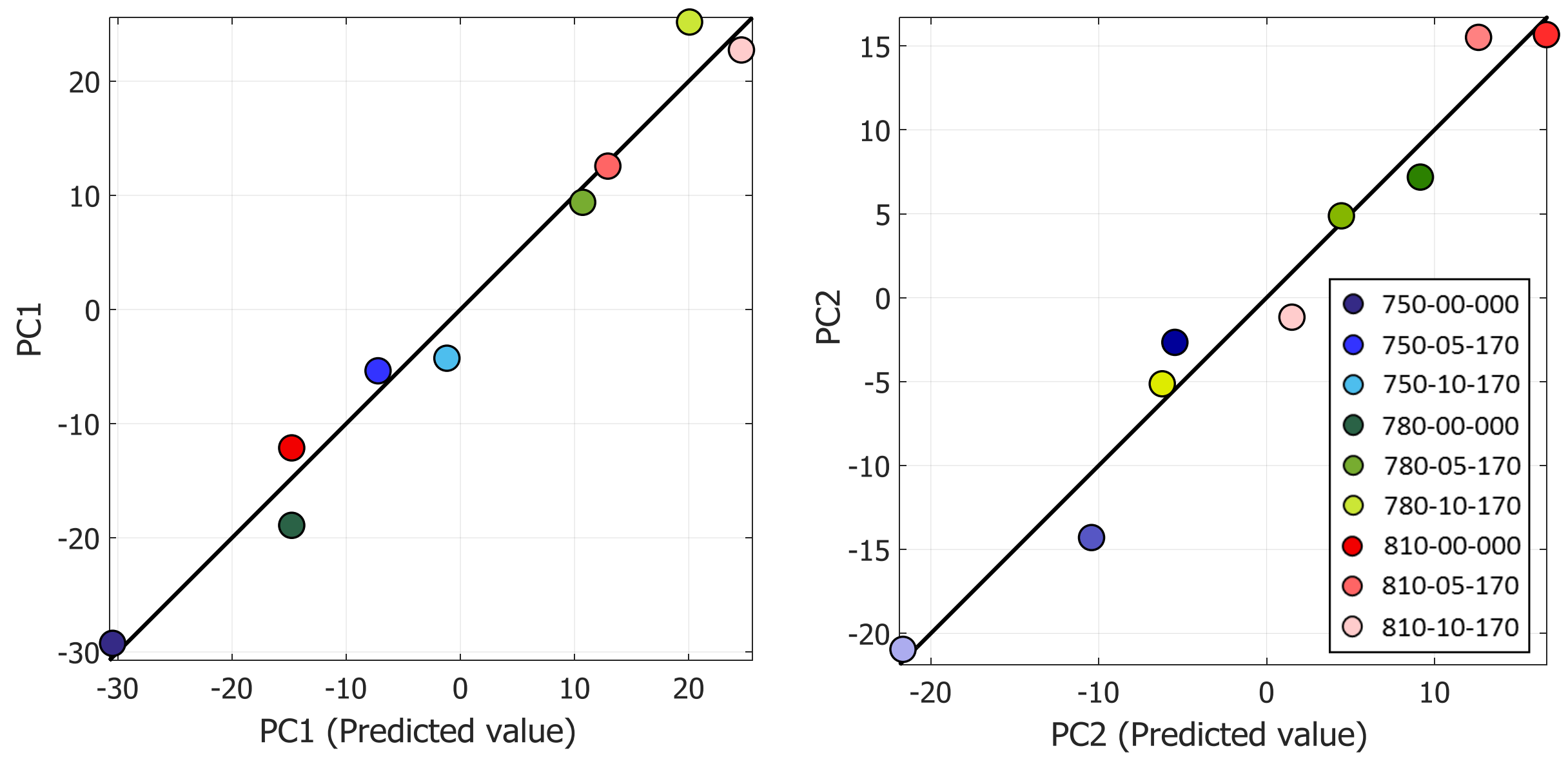

Figure 10. The accuracy of process-structure linkages established in this work using data science approaches. The process variables included the intercritical annealing temperature $(T)$ in ${ }^{\circ} \mathrm{C}$ and the amount of cold work (percentage of thickness reduction) from rolling process. Each point is an ensemble average of 2-point statistics of all scans from one processing history. 

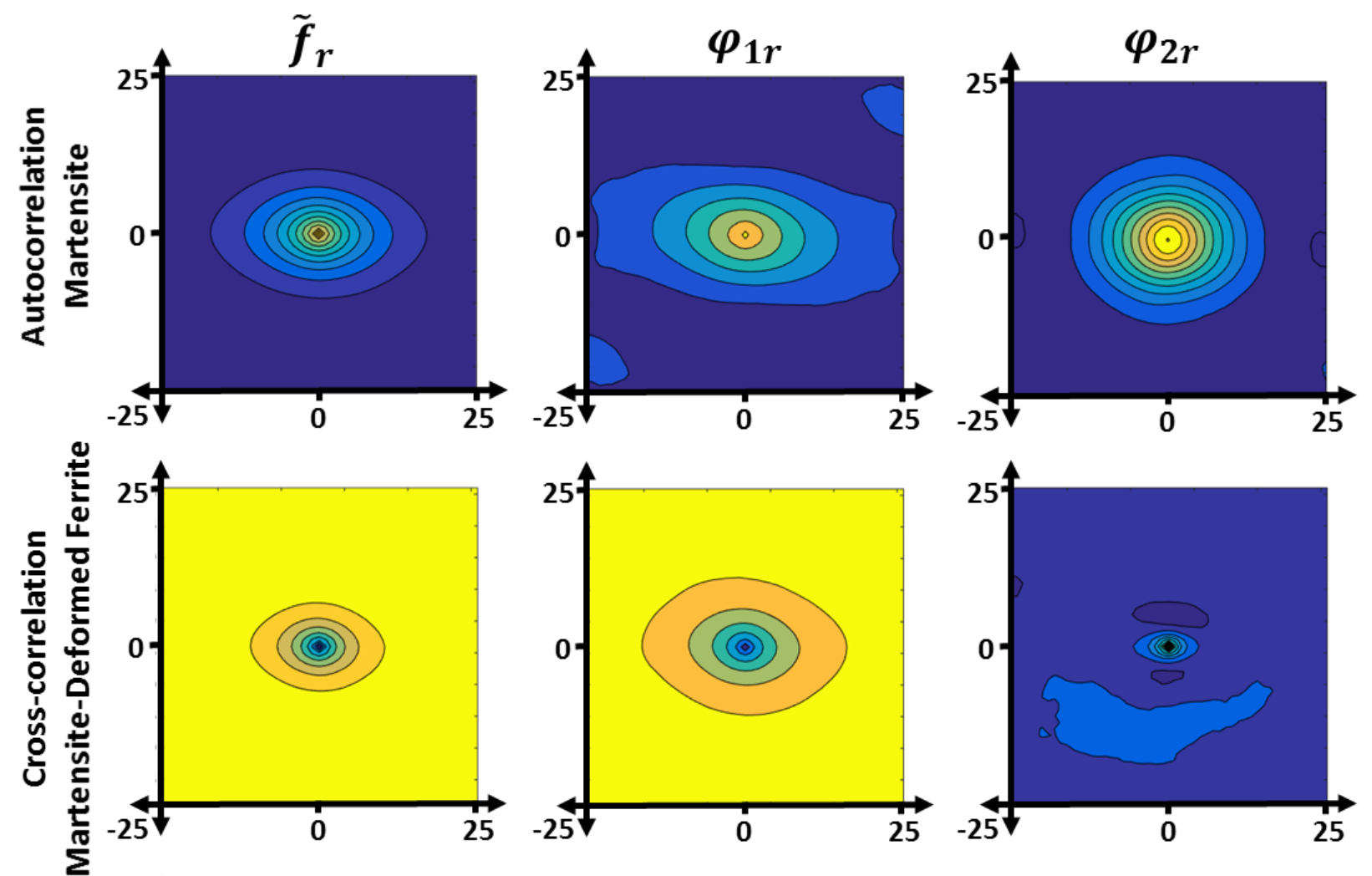

Probability
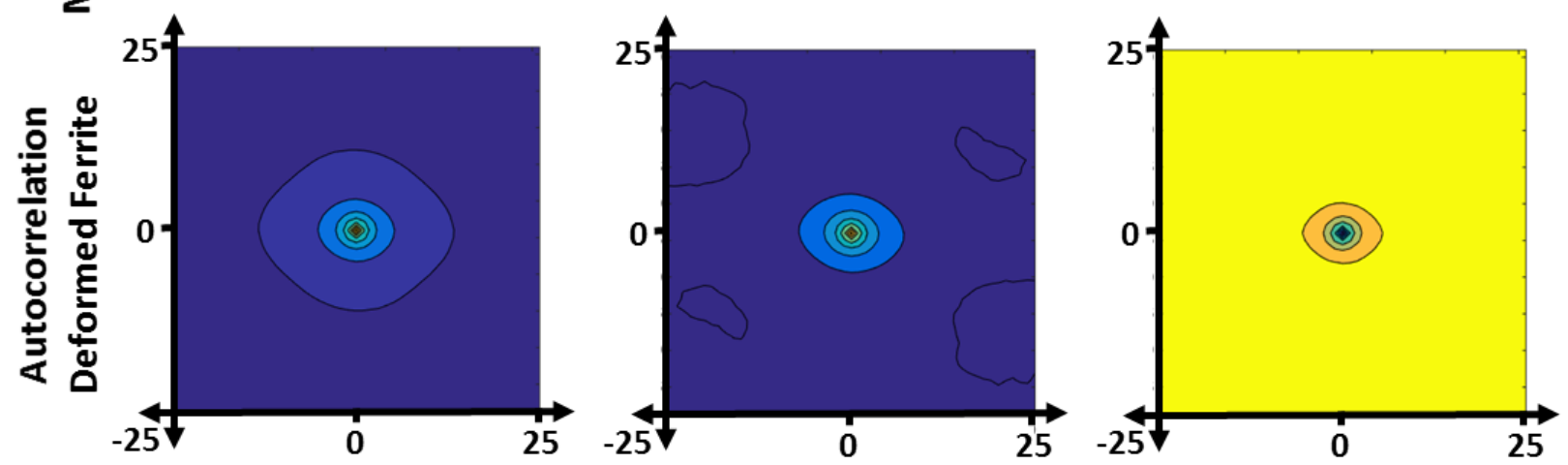

Low

Probability

Figure 11. The ensemble average and the first two PC basis $\left(\varphi_{1 r}\right.$ and $\varphi_{2 r}$; see Eq. (11)) computed in the PCA on the entire ensemble of microstructures studied. 


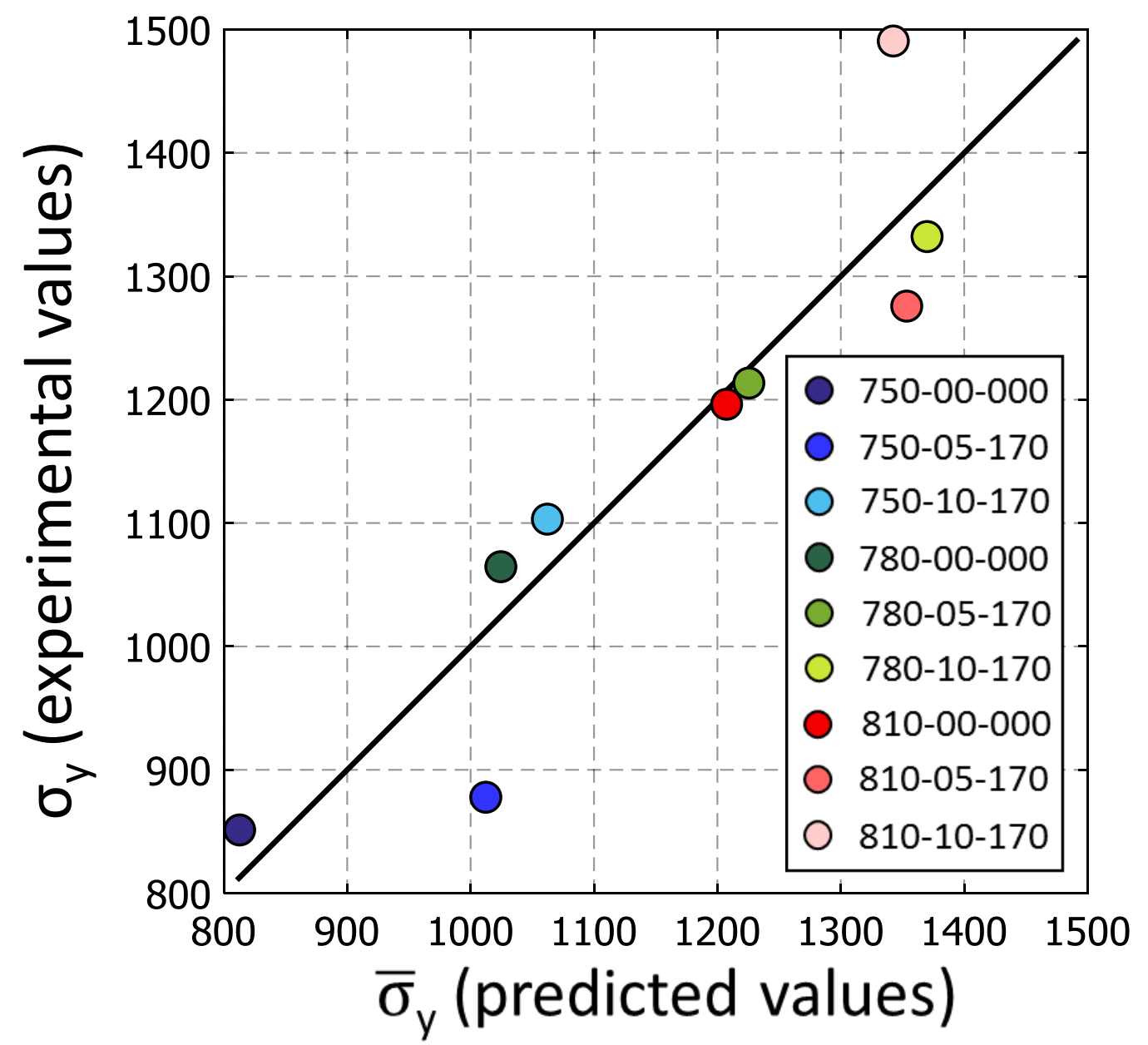

Figure 12. The accuracy of the structure-property linkage established in this work using data science approaches. The property captured is the indentation yield strength. Each point is an ensemble average of all measurements from one processing history. 
Table 1. Sample library and the labelling scheme employed in this study. The label includes information on intercritical annealing temperature, amount of cold work. and bake hardening temperature.

\begin{tabular}{|c|c|c|c|}
\hline Sample code & $\begin{array}{c}\text { Intercritical annealing } \\
\text { temperature }\left({ }^{\circ} \mathbf{C}\right)\end{array}$ & $\begin{array}{c}\text { Cold work -Thickness } \\
\text { reduction (\%) }\end{array}$ & $\begin{array}{c}\text { Bake hardening at } \\
\mathbf{1 7 0}{ }^{\circ} \mathbf{C} \text { for } \mathbf{2 0} \text { min }\end{array}$ \\
\hline $750-00-000$ & 750 & 0 & $\times$ \\
\hline $750-05-170$ & 750 & 5 & $\checkmark$ \\
\hline $750-10-170$ & 750 & 10 & $\checkmark$ \\
\hline $780-00-000$ & 780 & 0 & $\checkmark$ \\
\hline $780-05-170$ & 780 & 5 & $\checkmark$ \\
\hline $780-10-170$ & 780 & 10 & $\times$ \\
\hline $810-00-000$ & 810 & 0 & $\checkmark$ \\
\hline $810-05-170$ & 810 & 5 & $\checkmark$ \\
\hline $810-10-170$ & 810 & 10 & \\
\hline
\end{tabular}


Table 2. Comparison of the indentation yield strength, the indentation elastic modulus, and the contact area at yield point between samples subjected to different thermomechanical treatments. At least ten measurements were made on each sample.

\begin{tabular}{|c|c|c|c|}
\hline Sample code & $\begin{array}{c}\text { Average indentation } \\
\text { yield strength (MPa) }\end{array}$ & $\begin{array}{c}\text { Average indentation } \\
\text { elastic modulus (GPa) }\end{array}$ & $\begin{array}{c}\text { Radius of the contact } \\
\text { area at yield point }(\mu \mathrm{m})\end{array}$ \\
\hline $750-00-000$ & $899.5 \pm 62.9$ & $174.5 \pm 25.3$ & $142.3 \pm 7.0$ \\
\hline $750-05-170$ & $950.4 \pm 29.7$ & $193.4 \pm 23.3$ & $166.7 \pm 13.2$ \\
\hline $750-10-170$ & $1100.9 \pm 130.3$ & $200.9 \pm 22.3$ & $176.2 \pm 11.5$ \\
\hline $780-00-000$ & $1097.7 \pm 41.6$ & $216.8 \pm 23.3$ & $165.7 \pm 14.8$ \\
\hline $780-05-170$ & $1299.4 \pm 68.7$ & $209.6 \pm 15.4$ & $178.1 \pm 17.7$ \\
\hline $780-10-170$ & $1336.3 \pm 69.5$ & $201.9 \pm 16.7$ & $181.3 \pm 14.2$ \\
\hline $810-00-000$ & $1168.6 \pm 186.8$ & $188.5 \pm 14.7$ & $153.3 \pm 10.9$ \\
\hline $810-05-170$ & $1340.4 \pm 130.9$ & $219.2 \pm 14.5$ & $169.3 \pm 10.2$ \\
\hline $810-10-170$ & $1506.5 \pm 132.3$ & $207.6 \pm 9.7$ & $180.5 \pm 15.1$ \\
\hline
\end{tabular}



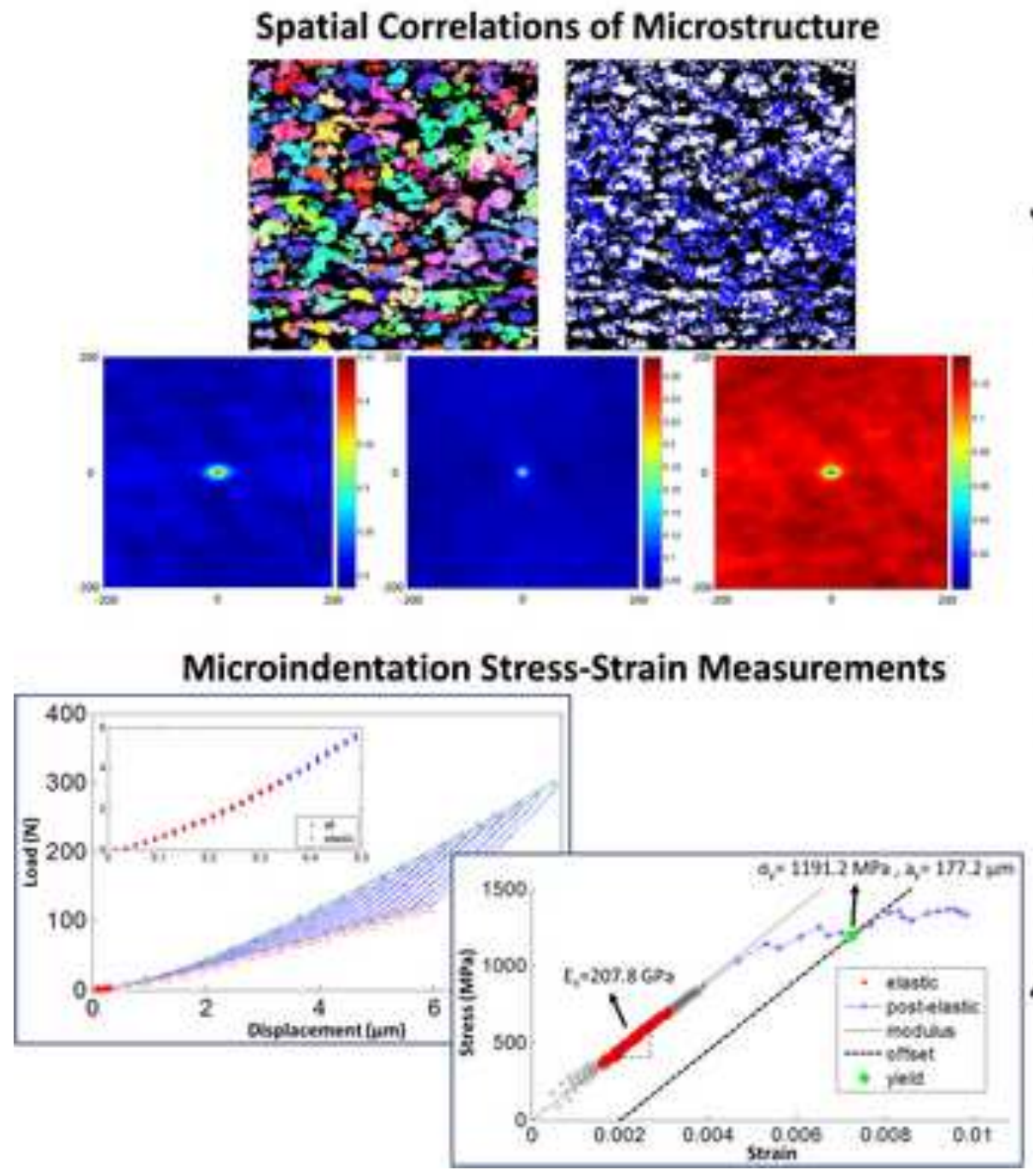

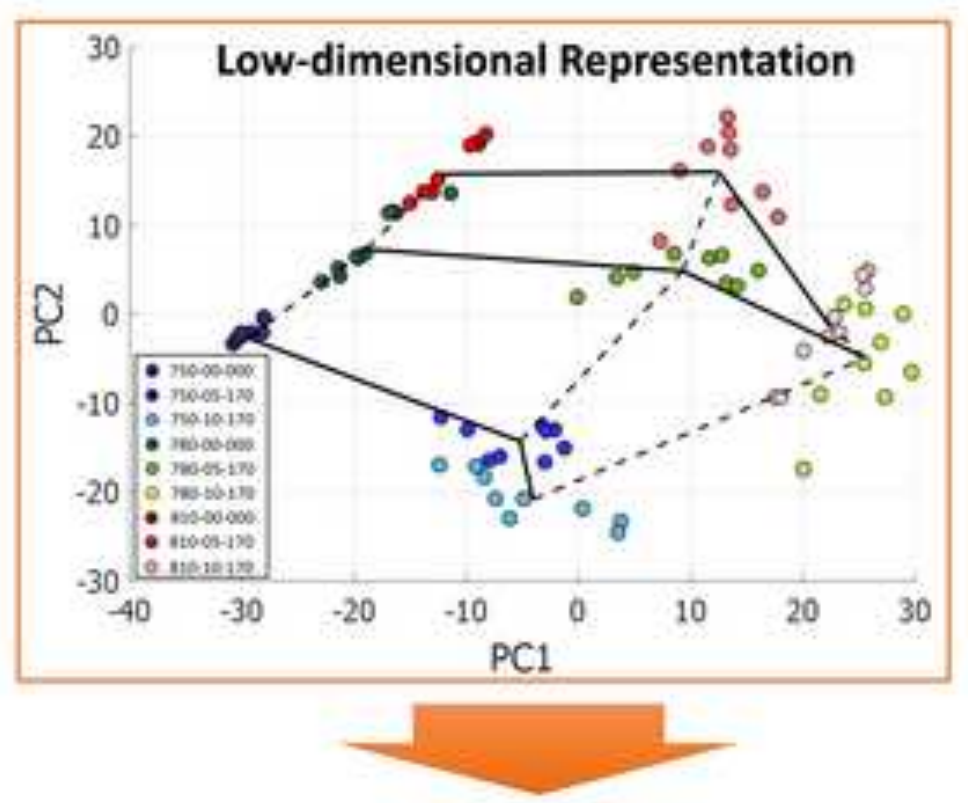

Process-structure-property (PSP) linkages
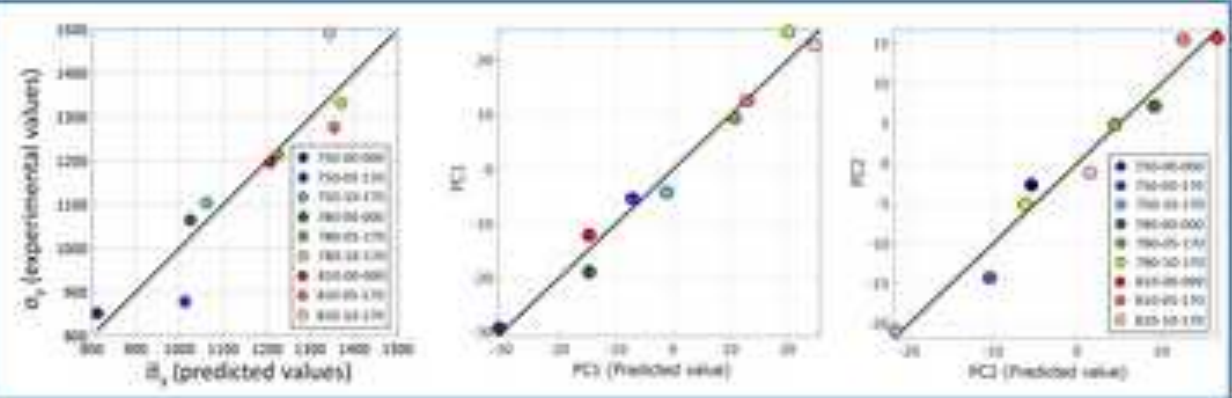